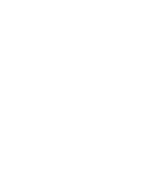
ANNUAL Further
REVEWS Furthe

Click here for quick links to Annual Reviews content online, including:

- Other articles in this volume

- Top cited articles

- Top downloaded articles

- Our comprehensive search

\section{Tuned Range-Separated Hybrids in Density Functional Theory}

\section{Roi Baer, ${ }^{1}$ Ester Livshits, ${ }^{1}$ and Ulrike Salzner ${ }^{2}$}

${ }^{1}$ Fritz Haber Center for Molecular Dynamics, Institute of Chemistry, The Hebrew University of Jerusalem, Jerusalem 91904 Israel; email: roi.baer@huji.ac.il

${ }^{2}$ Department of Chemistry, Bilkent University, 06800 Bilkent, Ankara, Turkey
Annu. Rev. Phys. Chem. 2010. 61:85-109

First published online as a Review in Advance on October 23, 2009

The Annual Review of Physical Chemistry is online at physchem.annualreviews.org

This article's doi:

10.1146/annurev.physchem.012809.103321

Copyright (c) 2010 by Annual Reviews. All rights reserved

0066-426X/10/0505-0085\$20.00

\section{Key Words}

time-dependent density functional theory, self-interaction, charge-transfer excitations, ionization potentials, Rydberg states
Abstract
We review density functional theory (DFT) within the Kohn-Sham (KS) and the generalized KS (GKS) frameworks from a theoretical perspective for both time-independent and time-dependent problems. We focus on the use of range-separated hybrids within a GKS approach as a practical remedy for dealing with the deleterious long-range self-repulsion plaguing many approximate implementations of DFT. This technique enables DFT to be widely relevant in new realms such as charge transfer, radical cation dimers, and Rydberg excitations. Emphasis is put on a new concept of system-specific range-parameter tuning, which introduces predictive power in applications considered until recently too difficult for DFT. 


\section{INTRODUCTION}

Density functional theory (DFT):

a framework for the electronic structure of matter; the key element is the electron density, not the wave function

Kohn-Sham (KS) method: an approach to apply DFT to electrons in a physical system by mapping it onto a system of noninteracting particles with the same density

Generalized Kohn-Sham (GKS) method: an approach to apply DFT to electrons in a physical system by mapping it onto a GKS system of particles obeying orbital nonlinear Schrödinger equations

TDDFT: timedependent density functional theory

Range-separated hybrid ( $\mathrm{RSH}$ ): a certain type of correction built into local density approximation that eliminates the dominant $(1 / r)$ offending part of the spurious long-range self-repulsion

MP: minimum principle
"To love practice without theory is like the sailor who boards ship without rudder and compass and is forever uncertain where he may cast." (Leonardo da Vinci, Notebook I, ca. 1490)

Leonardo's compass is indeed a simple and small device offering useful accuracy for reliable navigation in the turmoil of the oceans; quantum chemical methods based on density functional theory (DFT) (1-5) are approaching such a status. They are handy, relatively fast, and usefully accurate; they are intuitive and easy to interpret. Their impact on a broad variety of scientific fields, such as chemistry, condensed matter physics, and biology, is large and accelerating (6-11). Most of the impact of DFT has been delivered using the Kohn-Sham (KS) (12) and generalized KS (GKS) (13) orbital approaches, although orbital-less DFT is steadily developing (14-16). Whereas DFT applications are mostly used for the ground electronic state, an exceptionally successful offspring, the time-dependent DFT (TDDFT) $(17,18)$, enables access to excited states and dynamical electronic processes, such as molecular spectroscopy and photochemistry, intense lasers, and molecular electronics (19-34).

Despite this general success, the usual KS approximations go astray in some applications, producing qualitatively wrong predictions due to spurious self-repulsion. This has been seen in DFT (35-37) and TDDFT $(38,39)$. Although sophisticated methods in KS theory can be used for treating self-repulsion (40-42), an alternative and successful approach is the use of GKS theory combined with range-separated hybrids (RSHs) (43-47). Recent developments show that RSHs enable general, robust, consistent, and accurate remedies for self-repulsion (48-54).

This review presents an (arguably) systematic and rigorous way for RSHs as a DFT and a TDDFT. We focus on a relatively new notion, namely the ab initio-motivated tuning of the range parameter and its importance for gaining predictive power in many types of calculations; several theoretical and computational results are included for demonstration.

\section{HYBRID APPROACHES IN DENSITY FUNCTIONAL THEORY}

\section{A Generalized Kohn-Sham Approach to Density Functional Theory}

In this section, we describe the GKS approach with which we formulate RSHs as DFT approximations. The GKS concept (13) is extremely flexible, and there are many possibilities for its use; here we single out a natural but by no means canonical thread and for simplicity refer to it as our GKS approach.

Let us consider a system of $N$ electrons in a molecule with clamped nuclei [the BornOppenheimer (BO) approximation]. The Hamiltonian for the electrons is $\hat{H}=\hat{T}+\hat{U}+\hat{V}$, where $\hat{T}=\sum_{n=1}^{N}\left(-\frac{1}{2} \nabla_{n}^{2}\right)$ is the kinetic energy, $\hat{U}=\frac{1}{2} \sum_{n \neq m}^{N}\left(\frac{1}{r_{n m}}\right)$ is the electron-electron repulsion potential energy, and $\hat{V}=\int v(\mathbf{r}) \hat{n}(\mathbf{r}) d^{3} r$ is the potential energy of attraction to the nuclear charges (or other external potential fields), where $\hat{n}(\mathbf{r})=\sum_{n=1}^{N} \delta\left(\mathbf{r}-\hat{\mathbf{r}}_{n}\right)$ is the electron density operator. We may write the electronic ground-state energy as a minimum principle (MP),

$$
E_{g s}[v, N]=\min _{\Psi \rightarrow N}[\langle\Psi|\hat{H}| \Psi\rangle],
$$

searching over all normalized, antisymmetric $N$-electron wave functions $\Psi$. The minimizing wave function in Equation 1 is excruciatingly complicated, and the KS-DFT was introduced by Hohenberg \& Kohn (55) and Kohn \& Sham (12) to avoid direct reference to it. Levy (56) 
generalized their work by breaking the minimum procedure in Equation 1 into two stages:

$$
E_{g s}[v, N]=\min _{n \rightarrow N}\left[\min _{\Psi \rightarrow n}[\langle\Psi|\hat{T}+\hat{U}| \Psi\rangle]+\int n(\mathbf{r}) v(\mathbf{r}) d^{3} r\right] .
$$

Here $\Psi \rightarrow n$ means that the search is over all wave functions for which $\langle\Psi|\hat{n}(\mathbf{r})| \Psi\rangle=n(\mathbf{r})$, and $n \rightarrow N$ means that the search is over all (positive) density functions for which $\int n(\mathbf{r}) d^{3} r=N$. The inner minimum on the right-hand side defines a universal density functional,

$$
F[n]=\min _{\Psi \rightarrow n}[\langle\Psi|\hat{T}+\hat{U}| \Psi\rangle],
$$

where the minimizing wave function is denoted $\Psi_{*}$, and with it

$$
E_{g s}[v, N]=\min _{n \rightarrow N}\left[F[n]+\int n(\mathbf{r}) v(\mathbf{r}) d^{3} r\right] .
$$

The functional $F[n]$ is fantastically complicated, and we have no direct access to it; thus for practical calculations, it is beneficial to also define a simpler quantity, in which the minimum search is limited to $N$-electron Slater wave functions $\Phi$ (antisymmetric combinations of products of $N$ single-electron spin orbitals):

$$
F_{S}[n]=\min _{\Phi \rightarrow n}[\langle\Phi|\hat{T}+\hat{U}| \Phi\rangle],
$$

where the minimizing Slater wave function is denoted $\Phi_{*}$. The difference between the two functionals is the correlation energy (12):

$$
E_{C}^{G K S}[n]=F[n]-F_{S}[n] .
$$

Because of the simple structure of Slater wave functions, $F_{S}[n]$ is readily accessible; thus, $E_{C}^{G K S}[n]$ encapsulates the entire immensity of the electronic-structure problem, and it is this functional for which approximations must be crafted under DFT. Because $F_{S}$ involves a minimum search on a limited space, $F \leq F_{S}$, so $E_{C}^{G K S}[n]$ is always negative. One can now write the energy as

$$
E_{g s}[v, N]=\min _{n \rightarrow N}\left[F_{S}[n]+\int n(\mathbf{r}) v(\mathbf{r}) d^{3} r+E_{C}^{G K S}[n]\right] .
$$

Under several conditions described in Reference 13, we may replace this MP by

$$
E_{g s}[v, N]=\min _{\Phi \rightarrow N}\left[\langle\Phi|\hat{H}| \Phi\rangle+E_{C}^{G K S}\left[n_{\Phi}\right]\right],
$$

searching over all normalized Slater wave functions of $N$ orbitals, $\Phi$, and $n_{\Phi}(\mathbf{r})=\langle\Phi|\hat{n}(\mathbf{r})| \Phi\rangle$ is the corresponding electron density. This MP yields $\Phi_{*}$ as the minimizing Slater wave function. The two MPs (Equations 3 and 8) give the exact ground-state energy, and their minimizing wave functions have the same density:

$$
\left\langle\Phi_{*}|\hat{n}(\mathbf{r})| \Phi_{*}\right\rangle=\left\langle\Psi_{*}|\hat{n}(\mathbf{r})| \Psi_{*}\right\rangle .
$$

For applications, the MP in Equation 8 is written in terms of $N$ orthonormal spin orbitals $\phi_{j}\left(\mathbf{r}, s_{j}\right)$ $(j=1, \ldots, N),{ }^{1}$ with $\langle\Phi|\hat{T}| \Phi\rangle=\sum_{j=1}^{N}\left\langle\phi_{j}\left|-\frac{1}{2} \nabla^{2}\right| \phi_{j}\right\rangle,\langle\Phi|\hat{U}| \Phi\rangle=E_{H}\left[n_{\left\{\phi_{j}\right\}}\right]+E_{X}^{G K S}\left[\left\{\phi_{j}\right\}\right]$, where $n_{\left\{\phi_{j}\right\}}(\mathbf{r})=\sum_{j=1}^{N}\left|\phi_{j}(\mathbf{r})\right|^{2}, E_{H}[n]=\frac{1}{2} \iint \frac{n(\mathbf{r}) n\left(\mathbf{r}^{\prime}\right)}{\left|\mathbf{r}-\mathbf{r}^{\prime}\right|} d^{3} r d^{3} r^{\prime}$ is the Hartree energy, and

$$
E_{X}^{G K S}\left[\left\{\phi_{j}\right\}\right]=-\frac{1}{2} \iint \frac{\left|\sum_{j} \phi_{j}(\mathbf{r}) \phi_{j}\left(\mathbf{r}^{\prime}\right)\right|^{2}}{\left|\mathbf{r}-\mathbf{r}^{\prime}\right|} d^{3} r d^{3} r^{\prime}
$$

\footnotetext{
${ }^{1}$ For simplicity, we henceforth allow each electron to be in its own spatial spin orbital $\phi_{j}(\mathbf{r})$ of preassigned $z$ component of spin $s_{j}$ (up or down). Integrals involving two orbitals are zero if the spins of the two orbitals are not the same.
}

Correlation energy $\left(E_{C}\right)$ : the difference between the physical energy and the KS/GKS energy 
XC: exchange correlation

HF: Hartree-Fock is the orbital exchange. It is convenient to define the sum of exchange and correlation energies as the exchange-correlation (XC) orbital functional, $E_{X C}^{G K S}\left[\left\{\phi_{j}\right\}\right]=E_{X}^{G K S}\left[\left\{\phi_{j}\right\}\right]+E_{C}^{G K S}\left[n_{\left\{\phi_{j}\right\}}\right]$. The MP of Equation 8 thus becomes a search for $N$ normalized spin orbitals. Lagrange's minimum theory, with multipliers $\varepsilon_{j}$ to impose the normalization, produces the following GKS equations:

$$
\left(-\frac{1}{2} \nabla^{2}+v(\mathbf{r})+v_{H}(\mathbf{r})\right) \phi_{j}(\mathbf{r})+\hat{K}_{X} \phi_{j}(\mathbf{r})+v_{C}^{G K S}(\mathbf{r}) \phi_{j}(\mathbf{r})=\varepsilon_{j} \phi_{j}(\mathbf{r}),
$$

where

$$
\hat{K}_{X} \phi_{j}(\mathbf{r})=\frac{\delta E_{X}^{G K S}}{\delta \phi_{j}(\mathbf{r})}=-\sum_{k=1}^{N}\left[\phi_{k}(\mathbf{r}) \int \frac{\phi_{k}\left(\mathbf{r}^{\prime}\right) \phi_{j}\left(\mathbf{r}^{\prime}\right)}{\left|\mathbf{r}-\mathbf{r}^{\prime}\right|} d^{3} r^{\prime}\right], \quad v_{C}^{G K S}(\mathbf{r})=\frac{\delta E_{C}^{G K S}}{\delta n(\mathbf{r})} .
$$

The forms of the exchange functional and operator are identical to those appearing in HartreeFock (HF) theory.

In contrast to GKS, the $\mathrm{XC}$ energy in the $\mathrm{KS}$ approach is not an orbital functional, but a density functional: $E_{X C}^{K S}=E_{X}^{K S}+E_{C}^{K S}=F-T_{S}-E_{H}$, where $T_{S}[n]$ and $E_{X}^{K S}[n]$ are the kinetic and exchange energies, respectively, of a noninteracting electron system having the density $n(\mathbf{r})$ (the so-called KS system); by the Hohenberg-Kohn theorem, the KS system is uniquely defined. In terms of this, the Lagrange method yields the KS equations

$$
\left(-\frac{1}{2} \nabla^{2}+v(\mathbf{r})+v_{H}(\mathbf{r})+v_{X}(\mathbf{r})+v_{C}(\mathbf{r})\right) \phi_{j}(\mathbf{r})=\varepsilon_{j} \phi_{j}(\mathbf{r}),
$$

where $v_{l}(\mathbf{r})=\frac{\delta E_{l}^{K S}}{\delta n_{l}(\mathbf{r})}, l=H, X$, and $C$.

As we discuss below, the asymptotic $(r \rightarrow \infty)$ form of the potentials for finite systems is an important guide to constructing approximations, and it can be shown that to leading order in $r^{-1}$

$$
\begin{aligned}
& \mathrm{KS}: v_{X}(\mathbf{r}) \rightarrow-\frac{1}{r} ; \quad v_{C}(\mathbf{r}) \rightarrow v_{C}^{\infty}-\frac{\mathbf{r} \cdot \overleftrightarrow{\alpha} \cdot \mathbf{r}}{2 r^{6}}, \\
& \text { GKS: } \hat{K}_{X} \phi_{j}(\mathbf{r}) \rightarrow v_{X}(\mathbf{r}) \phi_{j}(\mathbf{r}) ; \quad v_{C}^{G K S}(\mathbf{r}) \rightarrow v_{C}^{K S}(\mathbf{r}),
\end{aligned}
$$

where $\overleftrightarrow{\alpha}$ is the polarizability tensor of the ionized system, and $v_{C}^{\infty}$ is an arbitrary constant, which we take as zero $(57,58)$. Clearly, in both approaches the asymptotic form of the XC potential is dominated by the exchange $-1 / r$ behavior.

The Hamiltonian in both the KS and GKS equations (Equations 13 and 11, respectively) is Hermitean, so the orbitals $\phi_{j}$ in each case are orthogonal. It is customary to index orbitals with ascending energies: $\cdots \leq \varepsilon_{j} \leq \varepsilon_{j+1} \leq \cdots(j=1,2, \ldots)$. Usually, only the first $N$ orbitals are needed (12), and these are the occupied orbitals; all other orbitals are unoccupied. Other orbital occupation rules are sometimes appropriate (see 59).

Both the KS and our GKS lead to the same ground-state density and energy, but the orbitals $\phi_{j}(\mathbf{r})$ and the orbital energies $\varepsilon_{j}$ are generally different, as are the Slater wave functions. In most KS approaches, one approximates each of the functionals $E_{X}^{K S}[n]$ and $E_{C}^{K S}[n]$ aiming at a good estimate of their sum $E_{X C}^{K S}[n]$ (although exact-exchange methods exist; see 60-62); in our GKS approach, we think of exchange as exact (Equation 10), whereas all other terms and approximations are loosely thought of as correlation energy. One should note that the difference between the KS and GKS XC energies is that of the kinetic energies: $E_{X C}^{K S}-E_{X C}^{G K S}=$ $\left\langle\Phi_{*}|\hat{T}| \Phi_{*}\right\rangle-T_{S}[n]$; this quantity is positive because $\langle\Phi|\hat{T}| \Phi\rangle \geq T_{S}[n]$ for any $\Phi \rightarrow n$ (2). Thus, the GKS system has higher kinetic energy than that of the KS system, whereas its XC energy is lower. 


\section{Some Constraints on the Kohn-Sham/Generalized Kohn-Sham Approaches}

Most KS approaches construct approximations for $E_{X C}^{K S}[n]$ using the great body of formal and numerical information existing for the infinite homogeneous electron gas (HEG); this leads to the local density approximation (LDA), as discussed below, as well as to various semilocal density approximations. A different source of information concerns the asymptotic long-range form of the XC potentials of finite systems (given in Equation 14) and the corresponding properties of the orbitals and density. In a system of $M$ interacting electrons, the density decays as $n(\mathbf{r}) \approx$ $e^{-2 \sqrt{2 \operatorname{IP}(M)} r}$, where $\operatorname{IP}(M)$ is the ionization potential (and all quantities are in atomic units) (63, 64). In HF theory, $n(\mathbf{r}) \approx e^{-2 \sqrt{-2 \varepsilon_{M}(M)} r}(58)$, where $\varepsilon_{M}(M)$ is the orbital energy of the highest occupied molecular orbital (HOMO). The arguments of Reference 58 hold for GKS as well. Because the physical and GKS systems have the same electron density (Equation 9), we find, by equating the density decay constants $(57,65)$,

$$
-\varepsilon_{M}(M)=\operatorname{IP}(M) \equiv E_{g s}(M-1)-E_{g s}(M) .
$$

[For improved readability, we replace the notation $E_{g s}[v, N]$ by $E_{g s}(N)$.] This important relation is the IP theorem connecting GKS quantities related to different charge states of the system. In HF theory, $-\varepsilon_{M}(M)$ is not the exact ionization energy, but in GKS the presence of correlation allows Equation 15 to hold exactly.

To obtain further information on the energy and DFT orbital energies, we follow Reference 65 and consider a generalization of the MP in Equation 8 to noninteger electron numbers $N=$ $M+\omega$, where $M$ is an integer and $0 \leq \omega \leq 1$. The search for a minimum is now over mixed-state density matrices, each comprising wave functions with $M$ and $M+1$ electrons. In this case, $N$ is the ensemble average of the number of electrons, and it is imposed on the ensemble MP (66) by a Lagrange multiplier. The resulting minimum energy $E_{g s}(N)$ is the linear average of the pure-state integer-number energies (65):

$$
E_{g s}(N)=E_{g s}(M+1) \omega+E_{g s}(M)(1-\omega) .
$$

The slope $E_{g s}^{\prime}(N)$ is the chemical potential $\mu$, and one finds by differentiation

$$
\begin{aligned}
& -\mu(M+\omega)=E_{g s}(M)-E_{g s}(M+1) \equiv \mathrm{EA}(M), \\
& -\mu(M-\omega)=E_{g s}(M-1)-E_{g s}(M) \equiv \operatorname{IP}(M) .
\end{aligned}
$$

The difference in the slope below $M$ and above it is called the fundamental gap: $\Delta \mu \equiv \mu(M+\omega)-$ $\mu(M-\omega)$. Note that the right-hand side of Equation 17 as well as the fundamental gap are both independent of $\omega$. By a generalization of a theorem due to Janak (67), the left slope of $E_{g s}$ also obeys $E_{g s}^{\prime}(M-\delta \omega)=\varepsilon_{M}(M)$ (where $\delta \omega$ is infinitesimal). This shows that the IP theorem (Equation 15) is a direct consequence of the second line of Equation 17. Furthermore, naively using Janak's theory for $M+\delta \omega$, we find $\mu(M+\delta \omega)=\varepsilon(M)_{M+1}$. Thus, the fundamental gap seems to give $\Delta \mu=\varepsilon(M)_{M+1}-\varepsilon(M)_{M}$. These arguments suggest that the fundamental gap $\Delta \mu$ is equal to the GKS (or KS) HOMO-LUMO gap $\Delta \mu_{G K S}=\varepsilon(M)_{M+1}-\varepsilon(M)_{M}$. Surprisingly, however, this result is wrong! In fact, exact KS calculations on small systems indicate that $\Delta \mu_{K S}<\Delta \mu$. This paradox is a result of the flawed use of Janak's theorem, which implicitly assumed that the correlation potential, when going through an integer number of electrons (from $M-\delta \omega$ to $M+\delta \omega$ ), is left unchanged. In fact, when an infinitesimal amount of electron charge moves the number of electrons from slightly below an integer to slightly above it, this potential must jump by a finite constant called the derivative discontinuity; anything different than a constant would violate the HK theorem (13), which demands uniqueness of the potential up to a constant. Because the gap
HEG: homogeneous electron gas

Local density approximation

(LDA): an approximate expression for the correlation energy based on quantities and concepts taken from the HEG

Ionization potential (IP) theorem: the energy needed to remove an electron from a system of $N$ electrons (to infinity) is equal exactly to the HOMO energy in the KS and GKS descriptions of this same system

Derivative discontinuity: the difference between the gap in the physical system and that in the $\mathrm{KS} / \mathrm{GKS}$ system 
Long-range self-repulsion: the spurious energy incurred by incomplete cancellation of the long-range Hartree energy by an approximate exchange energy functional in terms of orbital energies is obtained by combining Equations 15 and 17,

$$
\Delta \mu=\varepsilon(M+1)_{M+1}-\varepsilon(M)_{M},
$$

we find the following relation between the fundamental and GKS gaps:

$$
\Delta \mu=\Delta \mu_{G K S}+\varepsilon(M+1)_{M+1}-\varepsilon(M)_{M+1},
$$

showing that the derivative discontinuity is the difference between the HOMO energy of the $M+1$ electron system and the LUMO energy of the $M$ electron system.

\section{Local Density Approximation in our Generalized Kohn-Sham Eyes}

Our GKS equations (Equation 11) can be used to find the approximate ground-state energy and density of any electronic system based on the availability of simple and effective approximations for $E_{C}$, for which the LDA is the cornerstone approach (12). The LDA originates from approximating the sum of the XC energy by local (Thomas-Fermi type) energy expressions:

$$
E_{X C}^{K S}[n] \approx E_{X C}^{L D A-K S}[n]=\int \varepsilon_{X}^{H E G}(n(\mathbf{r})) n(\mathbf{r}) d^{3} r+\int \varepsilon_{C}^{H E G}(n(\mathbf{r})) n(\mathbf{r}) d^{3} r,
$$

where $\varepsilon_{X}^{H E G}(n)$ and $\varepsilon_{C}^{H E G}(n)$ are the exchange and correlation energies per electron in a HEG, respectively. $\varepsilon_{X}^{H E G}(n)$ is evaluated by an analytical expression, whereas for $\varepsilon_{C}^{H E G}(n)$, simple approximate expressions exist (68-70). By construction, Equation 20 holds exactly for homogeneous densities. For the inhomogeneous case, the integrals over $n \varepsilon_{l}(n)$ are crude approximations to $E_{l}[n](l=X, C)$. However, the $X$ and $C$ errors tend to cancel when added (3), so the sum is often a useful approximation to $E_{X C}^{K S}[n]=E_{X}^{K S}[n]+E_{C}^{K S}[n]$. The KS equations (Equation 13) using the LDA functionals are

$$
-\frac{1}{2} \nabla^{2} \phi_{j}(\mathbf{r})+\left(v(\mathbf{r})+v_{H}(\mathbf{r})+v_{X}^{K S, L D A}(n(\mathbf{r}))+v_{C}^{K S, L D A}(n(\mathbf{r}))\right) \phi_{j}(\mathbf{r})=\varepsilon_{j} \phi_{j}(\mathbf{r}),
$$

with

$$
v_{H}[n](\mathbf{r})=\int \frac{n\left(\mathbf{r}^{\prime}\right)}{\left|\mathbf{r}-\mathbf{r}^{\prime}\right|} d^{3} r^{\prime}, \quad v_{l}^{K S, L D A}(n)=\left(n \varepsilon_{l}^{H E G}(n)\right)^{\prime}, \quad l=X, C,
$$

as the Hartree and LDA exchange and correlation potentials. From the above discussion, the KSLDA XC potential $v_{X C}^{K S, L D A}(\mathbf{r})=v_{X}^{K S, L D A}(n(\mathbf{r}))+v_{C}^{K S, L D A}(n(\mathbf{r}))$ still has the wrong asymptotic form as it decays exponentially with $r \rightarrow \infty$ instead of as $-r^{-1}$ as demanded by Equation 14 . We can see this also by rewriting Equation 20 in a GKS way as follows:

$$
\begin{aligned}
E_{X C}^{L D A-G K S}\left[\left\{\phi_{j}\right\}\right]= & E_{X}^{G K S}\left[\left\{\phi_{j}\right\}\right]+\int \varepsilon_{C}^{H E G}(n(\mathbf{r})) n(\mathbf{r}) d^{3} r \\
& +\left[\int \varepsilon_{X}^{H E G}(n(\mathbf{r})) n(\mathbf{r}) d^{3} r-E_{X}^{G K S}\left[\left\{\phi_{j}\right\}\right]\right] .
\end{aligned}
$$

The first term on the right-hand side gives the asymptotic potential required by Equation 14 . If we think of the last three terms on the right as a kind of correlation energy, we find that the correlation-energy functional gradient acts, for $r \rightarrow \infty$, as a repulsive $1 / r$ Coulomb potential in stark contradiction to the condition for $v_{C}^{G K S}(\mathbf{r})$ in Equation 14. We demonstrate the spurious behavior of the potentials in LDA in Figure 1; some grossly incorrect predictions of electronic structure and dynamics by LDA are attributed to it $(37,41,71-76)$.

A similar analysis can be made for the generalized gradients approximation (GGA) and other semilocal functionals that use expressions of the form $\int g_{X / C}(n(\mathbf{r}),|\nabla n(\mathbf{r})|) d^{3} r(77-79)$. Within GGA, attempts to enforce the $-1 / r$ dependency into $v_{X C}^{s l}$ led to significantly improved functionals (79), but the asymptotic form of the potential was still spurious (80). 
a

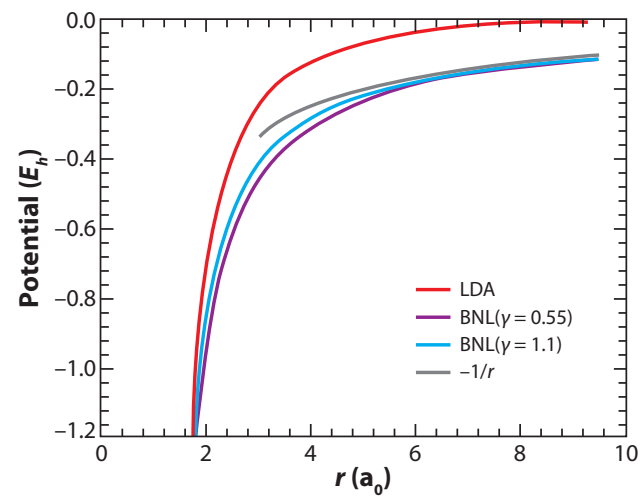

b

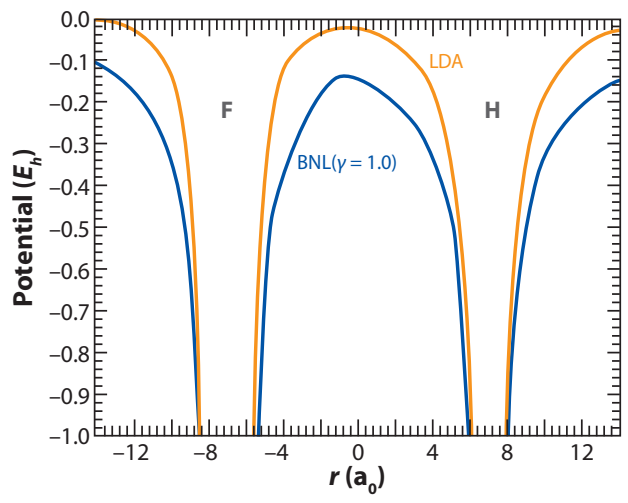

Figure 1

(a) The Kohn-Sham local density approximation (KS LDA) potential tail and two average generalized Kohn-Sham/Baer-Neuhauser-Livshits (GKS/BNL) potential tails for the Ar atom (located at $r=0$ ). The $-1 / r$ Coulomb potential tail is also shown. (b) The KS LDA potential and average GKS/BNL potentials of a $\mathrm{F}$...H system ( $14 \mathrm{a}_{0}$ apart) along the internuclear axis.

\section{Scaled Hybrids}

The scaled-hybrid (SH) approach (81-83) helps to mitigate the spurious self-repulsion appearing in the square brackets of Equation 23 by scaling that term, multiplying it by a factor $0<\gamma<1$ :

$$
\begin{aligned}
E_{X C}^{S H-L D A}\left[\left\{\phi_{j}\right\}\right]= & E_{X}^{G K S}\left[\left\{\phi_{j}\right\}\right]+\int \varepsilon_{C}^{H E G}(n(\mathbf{r})) n(\mathbf{r}) d^{3} r \\
& +\gamma\left[\int \varepsilon_{X}^{H E G}(n(\mathbf{r})) n(\mathbf{r}) d^{3} r-E_{X}^{G K S}\left[\left\{\phi_{j}\right\}\right]\right] .
\end{aligned}
$$

We can think of the last three terms in Equation 24 as a kind of correlation energy and again find that it has the wrong asymptotic energy gradient at large $r$, namely the self-repulsive $\frac{\gamma}{r}$ potential. A benefit of the SH approach over LDA is that this repulsive potential is reduced by the scaling factor $\gamma$. Obviously, a similar treatment applies using semilocal functionals.

The scaling parameter $\gamma$ is determined semiempirically, by calibrating to known molecular atomization energies, IPs, proton affinities, and total atomic energies. The semiempirical procedure places the recommended value of $\gamma$ between 0.5 and $0.8(81,84)$. Some applications of this approach determine $\gamma$ from theoretical arguments $(82,85)$. The $\mathrm{SH}$ approach spawned several successful functionals $(83,85-87)$, especially for the prediction of the ground-state potential surface near its minima (bond lengths, atomization energies, vibrational frequencies). We note, however, that the residual self-repulsion still leads to well-known failures of this method, as shown in several examples below.

\section{Range-Separated Hybrids}

Another way to mitigate the spurious long-range behavior of LDA (and other semilocal XCenergy functionals) is to damp the long-range orbital exchange energy term appearing in the square brackets of the LDA XC energy (Equation 23) complementing it with a matching local

Scaled hybrid (SH): a certain type of correction built into LDA or GGA that mitigates the spurious long-range selfrepulsion 
BNL: Baer-

Neuhauser-Livshits RSH functional

exchange functional $(43,88)$. This is the RSH approach, and it leads to the following XC functional:

$$
E_{X C}^{R S H-G K S}\left[\left\{\phi_{j}\right\}\right]=E_{X}^{G K S}\left[\left\{\phi_{j}\right\}\right]+\int \varepsilon_{C}^{\gamma}(n(\mathbf{r})) n(\mathbf{r}) d^{3} r+\left[\int \varepsilon_{X}^{\gamma-H E G}(n(\mathbf{r})) n(\mathbf{r}) d^{3} r-E_{X}^{\gamma}\left[\left\{\phi_{j}\right\}\right]\right],
$$

where $E_{X}^{\gamma}$ is the following orbital exchange functional,

$$
E_{X}^{\gamma}\left[\left\{\phi_{j}\right\}\right]=-\frac{1}{2} \iint\left|\sum_{j} \phi_{j}(\mathbf{r}) \phi_{j}\left(\mathbf{r}^{\prime}\right)\right|^{2} y_{\gamma}\left(\left|\mathbf{r}-\mathbf{r}^{\prime}\right|\right) d^{3} r d^{3} r^{\prime},
$$

corresponding to a short-range, screened, electron interaction, for example, the Yukawa kernel (47, 89) $y_{\gamma}(r)=\frac{e^{-\gamma r}}{r}$ or the erfc kernel $(44,88) y_{\gamma}(r)=\frac{\operatorname{erfc}(\gamma r)}{r}$. The complementary local exchange energy $\varepsilon_{X}^{\gamma-H E G}(n)$ is given in Reference 90 for the Yukawa kernel and in Reference 44 for the erfc kernel, and the choice $\varepsilon_{C}^{\gamma}(n)=\varepsilon_{C}^{H E G}(n)$ yields a functional fully consistent with the HEG. Comparing Equation 25 to Equation 23, it is evident that the long-range Coulomb repulsion energy in the square brackets of the latter is missing in the former; i.e., the RSH XC potentials display the correct $-1 / r$ attractive form, as required by Equation 14 .

The RSH-GKS equations resulting from minimization of the energy functional (Equation 11) are

$$
\left(-\frac{1}{2} \nabla^{2}+v(\mathbf{r})+v_{H}(\mathbf{r})+v_{X}^{\gamma}(\mathbf{r})+v_{C}^{\gamma}(\mathbf{r})\right) \phi_{j}(\mathbf{r})+\hat{K}_{X}^{\gamma} \phi_{j}(\mathbf{r})=\varepsilon_{j} \phi_{j}(\mathbf{r})
$$

with

$$
\begin{aligned}
\hat{K}_{X}^{\gamma} \phi_{j}(\mathbf{r}) & =\frac{\delta E_{X}^{\gamma}}{\delta \phi_{j}(\mathbf{r})}=-\sum_{k=1}^{N}\left[\phi_{k}(\mathbf{r}) \int \phi_{k}\left(\mathbf{r}^{\prime}\right) \phi_{j}\left(\mathbf{r}^{\prime}\right) \bar{y}_{\gamma}\left(\left|\mathbf{r}-\mathbf{r}^{\prime}\right|\right) d^{3} r^{\prime}\right] \\
v_{l}^{\gamma}(n) & =\left(\varepsilon_{l}^{\gamma}(n) n\right)^{\prime} \quad l=X, C,
\end{aligned}
$$

where $\bar{y}_{\gamma}(r)=\frac{1}{r}-y_{\gamma}(r)$ is the complementary interaction. We can observe the long-range effects of the RSH approach by inspecting the average potential (see the sidebar for a definition) and comparing it to the total (KS) potential of the LDA, as seen in Figure $\mathbf{1}$ for the Ar atom and for the F. .H system along the internuclear axis. We choose the Baer-Neuhauser-Livshits (BNL) $(47,52)$ form of the RSH (see below for more details on its structure). The KS/LDA potentials are tighter near their respective nuclei and decay faster to zero when vacuum is approached, whereas the GKS/ BNL average potential decays slowly as $-1 / r$. In the figure we repeat the RSH calculation with a different value of $\gamma$ (for the Ar atom) and find this affects only the potential near

\section{THE AVERAGE POTENTIAL}

The GKS equation contains orbital operators, such as $\hat{K}_{X}$, that are not local potentials, as in KS theory. It may be useful to describe these in terms of an approximate average potential $v_{a v g}(\mathbf{r})$. Starting from the set of converged RSH orbitals, $\phi_{j}(\mathbf{r}) j=1, \ldots, N$, the $v_{\text {avg }}(\mathbf{r})$ and the effective orbital energies $\varepsilon_{j}$ are determined by requiring minimal deviance from local Schrödinger equations. Thus, one defines the deviance $\left|D_{j}\right\rangle=\left(\varepsilon_{j}-\left[-\frac{1}{2} \nabla^{2}+v_{\text {avg }}\right]\right)\left|\phi_{j}\right\rangle$ and minimizes $L\left[v_{\text {avg }},\left\{\varepsilon_{j}\right\}\right]=\sum_{j=1}^{N}\left\langle D_{j} \mid D_{j}\right\rangle$, which results in the simultaneous equations

$$
v_{a v g}(\mathbf{r})=\frac{1}{n(\mathbf{r})} \sum_{j=1}^{N} \phi_{j}(\mathbf{r})\left(\varepsilon_{j}+\frac{1}{2} \nabla^{2}\right) \phi_{j}(\mathbf{r}), \quad \varepsilon_{j}=\left\langle\phi_{j}\left|v_{a v g}+\frac{1}{2} \nabla^{2}\right| \phi_{j}\right\rangle,
$$

where $\varepsilon_{j}$ and $v_{\text {avg }}(\mathbf{r})$ are determined up to a common $j$-independent constant. For orbitals coming from a molecular GKS, this constant can be chosen so that $\varepsilon_{N}$ is equal to the GKS HOMO energy. This should yield $v_{a v g}(\mathbf{r})$ approaching zero as $r \rightarrow 0$. 
the nucleus, but the asymptotic form is the same. The GKS/BNL asymptotic behavior is correct because it is in accord with Equation 14 (for overall neutral systems); furthermore, we found that KS/LDA predicts the separated atoms partially charged $\left(\mathrm{F}^{-0.2} \ldots \mathrm{H}^{+0.2}\right)$, whereas $\mathrm{GKS} / \mathrm{BNL}$ correctly predicts neutral atoms.

RSHs have also been realized using semilocal functionals (44, 46-53). In most implementations, $\gamma$ is determined as a universal constant as for the scaling parameter in SHs. The issue of tuning $\gamma$ on a system-specific basis is one focus of the present review and has not been widely addressed (but see Reference 91 for novel generalizations).

\section{Generalized Kohn-Sham Approach to Time-Dependent Density Functional Theory}

In time-dependent problems, molecular electrons are subject to time-dependent fields (e.g., laser pulses) described by external potentials $v(\mathbf{r}, t)$, and the Hamiltonian of the system is $\hat{H}(t)=$ $\hat{T}+\hat{U}+\hat{V}(t)$, where $\hat{V}(t)=\sum_{m} v\left(\mathbf{r}_{m}, t\right)$. TDDFT focuses on the space-time density $n(\mathbf{r}, t)$ and allows the construction of a TDKS scheme, replacing the correlated time-dependent wave function by a time-dependent Slater wave function obeying a time-dependent Schrödinger equation with a TDKS potential without electron-electron interaction.

The basic theorem, by Runge \& Gross (17), shows that such a construction is unique. More precisely, given the initial $(t=0)$ state $\Psi_{0}$, if $v_{1}(\mathbf{r}, t)$ and $v_{2}(\mathbf{r}, t)$ are two potentials inducing the same $n(\mathbf{r}, t)$, then they differ by at most a purely time-dependent function $c(t)$. This is also true for a system of noninteracting electrons, the KS system: Starting from a Slater wave function $\Phi_{0}$ (having the same initial density and current density as $\Psi_{0}$ ), there is a unique (up to a purely time-dependent constant) potential $v_{T D K S}(\mathbf{r}, t)$, which produces the time-dependent density $n(\mathbf{r}, t)$. This leads to the definition of a time-dependent exchange-correlation (TDXC) potential $(17,92)$ :

$$
v_{T D K S}(\mathbf{r}, t)=v(\mathbf{r}, t)+v_{H}[n(t)](\mathbf{r})+v_{T D X C}\left[\Psi_{0}, \Phi_{0}, n\right](\mathbf{r}, t),
$$

where $n(\mathbf{r}, t)=\sum_{j=1}^{N}\left|\phi_{j}(\mathbf{r}, t)\right|^{2}$, and the time-dependent Schrödinger equation for the KS system is

$$
i \dot{\phi}_{k}(\mathbf{r}, t)=\left(-\frac{1}{2} \nabla^{2}+v_{T D K S}(\mathbf{r}, t)\right) \phi_{k}(\mathbf{r}, t) .
$$

There is no rigorous route for constructing approximations for the universal TDXC potential. One guideline, appropriate when $\Psi_{0}$ and $\Phi_{0}$ are ground states with the same initial density, is the adiabatic theorem of quantum mechanics from which one deduces that for a slowly varying density $n(t)$, the effective potential is the instantaneous ground-state potential:

$$
v_{T D K S}[n](\mathbf{r}, t)=v_{K S}[n(t)](\mathbf{r}) .
$$

Using Equation 29, we find $v_{T D X C}[n](\mathbf{r}, t)=v_{X C}[n(t)](\mathbf{r})$. In the adiabatic approximation, this ansatz is used even when the density changes rapidly. Numerical (93) and theoretical (94) indications show that this approach can be reliable even for strongly nonadiabatic situations. In actual applications, one further approximates $v_{X C}[n]$ by their LDA, semilocal, or various SH replacements. Despite these uncontrolled approximations, surprisingly good results for excitation energies in many systems are found $(21,95-102)$. One possible reason for this is that within the adiabatic approximation Equation 30 is derivable from a stationary principle of the action

$$
S_{a d}[\Phi]=\int_{0}^{T_{f}}\left\{\left\langle\Phi(t)\left|i \frac{\partial}{\partial t}-\hat{H}(t)\right| \Phi(t)\right\rangle-E_{C}^{K S}\left[n_{\Phi(t)}\right]\right\} d t .
$$

Such a property guarantees the 0 -XC force condition and Galilean invariance (108). Terms of TDXC potentials beyond the adiabatic approach (103) are usually called memory effects (104) and 
are especially important for double excitations (105) and fast electron dephasing in metals (106). The inclusion of memory is next to impossible with local TDXC approximations to TDDFT because Galilean invariance and the 0 -force conditions cannot be imposed (107-109) as there is no stationary principle for nonadiabatic functionals in TDDFT (110). These problems prompted KS-like developments within time-dependent current-density functionals $(109,111,112)$, metric or deformation tensor functionals (106, 113-116), and potential-adaptation methods (117, 118).

Another problem with adiabatic semilocal functionals within TDKS theory is that they predict too low excitation energies for long-range charge-transfer excitations (CTEs) (38, 45, 52, 119, 120). We explain and expand on this problem below and show that the application of RSHs within TDGKS can mitigate the problems considerably.

We now discuss the TDGKS equations as a TDDFT approach. The TDGKS equations we consider are of the form

$$
i \dot{\phi}_{k}(\mathbf{r}, t)=\left(-\frac{1}{2} \nabla^{2}+v_{T D G K S}(\mathbf{r}, t)+\hat{W}\left[\left\{\phi_{j}\right\}\right]\right) \phi_{k}(\mathbf{r}, t),
$$

where $\hat{W}=\hat{J}+\hat{K}$, and the orbital operators are given by

$$
\begin{aligned}
\hat{J}\left[\left\{\phi_{j}\right\}\right] f(\mathbf{r}) & =\left(\int n\left(\mathbf{r}^{\prime}, t\right) j[n(t)]\left(\left|\mathbf{r}-\mathbf{r}^{\prime}\right|\right) d^{3} r^{\prime}\right) f(\mathbf{r}), \\
\hat{K}\left[\left\{\phi_{j}\right\}\right] f(\mathbf{r}) & =-\sum_{k=1}^{N}\left[\phi_{k}(\mathbf{r}) \int \phi_{k}\left(\mathbf{r}^{\prime}\right) f\left(\mathbf{r}^{\prime}\right) k[n(t)]\left(\left|\mathbf{r}-\mathbf{r}^{\prime}\right|\right) d^{3} r^{\prime}\right] .
\end{aligned}
$$

Here $j[n](r)$ and $k[n](r)$ can be any potential density functionals, not necessarily Coulomb. Obviously, this includes SH, RSH, and HF functionals. The TDKS equations were justified by the application of the Runge-Gross theorem to noninteracting electrons, but the TDGKS equations are of a different form, and a different proof is needed. It indeed is possible to prove that for a given initial set of orthonormal orbitals $\phi_{k}(\mathbf{r}, 0)(k=1, \ldots, N)$, if the potential $v_{T D G K S}(\mathbf{r}, t)$ generates a TD density $n(\mathbf{r}, t)$ through the TDGKS equation (Equation 33), and if it is Taylor expandable, then it is unique up to a time-dependent constant $c(t)$. The proof closely follows the standard Runge-Gross proof but relies on an additional (readily proven) lemma: that time propagation according to Equation 33 leads to a density and current density that obey the continuity relation.

The $v_{T D G K S}(\mathbf{r}, t)$ potential can now be approximated as follows:

$$
v_{T D G K S}(\mathbf{r}, t)=v(\mathbf{r}, t)+v_{H}(\mathbf{r}, t)+v_{T D X C}^{H Y B}(\mathbf{r}, t),
$$

where $v_{H}(\mathbf{r}, t)$ is the instantaneous Hartree potential (Equation 22), and the XC-Hyb potential $v_{T D X C}^{H Y B}(\mathbf{r}, t)$ is a local or semilocal adiabatic functional of the density. An attractive property of the TDGKS equations is that they can be derived from a stationary action principle, similar to Equation 32. Furthermore, the TDGKS equations include memory effects through the timedependent phases of the orbitals in the $W$ term. However, these memory effects do not come in the first-order response of the approach. We show below that the application of RSHs (as part of $\hat{W}$ ) within the TDGKS equations has several advantages over local adiabatic KS approaches.

Orbital functionals can be inserted into TDKS through time-dependent optimized effective potential methods $(42,121)$. Numerically, this approach is extremely demanding, and the timedependent Krieger-Li-Iafrate approach was developed and used in some applications (42, 122, 123). This, however, violates the $0-\mathrm{XC}$ force condition $(118,124)$.

\section{AB INITIO-MOTIVATED TUNING OF THE RANGE PARAMETER $\gamma$}

We now discuss the parameter $\gamma$ in SHs and RSHs. Our analysis sidesteps the adiabatic connection theorem in other works $(81,125-127)$. The correlation-energy functional was defined as the 
difference between two expectation values of the operator $\hat{T}+\hat{U}$ (Equation 6). It can also be written as a difference of two expectation values of a purely potential operator $\hat{Y}_{\gamma}=\frac{1}{2} \sum_{n \neq m} y_{\gamma}\left(r_{n m}\right)$, where $y_{\gamma}(r)$ is a potential having the following basic properties: It converges to a Coulomb repulsion at large $r$, and it is everywhere repulsive:

$$
\lim _{\gamma \rightarrow 0} y_{\gamma}(r)=\frac{1}{r}, \quad \lim _{\gamma \rightarrow \infty} y_{\gamma}(r)=0, \quad y_{\gamma}(r)>0, \quad y_{\gamma}^{\prime}(r)<0 .
$$

To be specific, we consider generic functions of the following type:

$$
\begin{aligned}
y_{\gamma}(r)=\frac{e^{-\gamma r}}{r} & \text { RSH-Yuk, } \\
y_{\gamma}(r)=\frac{\operatorname{erfc}(\gamma r)}{r} & \text { RSH-erfc, } \\
y_{\gamma}(r)=\frac{1}{1+\gamma a_{0}} \frac{1}{r} & \text { SH. }
\end{aligned}
$$

We note the following inequalities:

$$
\begin{aligned}
& \lim _{\gamma \rightarrow 0}\left[\left\langle\Psi_{*}\left|\hat{Y}_{\gamma}\right| \Psi_{*}\right\rangle-\left\langle\Phi_{*}\left|\hat{Y}_{\gamma}\right| \Phi_{*}\right\rangle\right]=\left\langle\Psi_{*}|\hat{U}| \Psi_{*}\right\rangle-\left\langle\Phi_{*}|\hat{U}| \Phi_{*}\right\rangle=E_{C}-T_{C} \leq E_{C}, \\
& \lim _{\gamma \rightarrow \infty}\left[\left\langle\Psi_{*}\left|\hat{Y}_{\gamma}\right| \Psi_{*}\right\rangle-\left\langle\Phi_{*}\left|\hat{Y}_{\gamma}\right| \Phi_{*}\right\rangle\right]=0 \geq E_{C} .
\end{aligned}
$$

The first is true because $T_{C}=\left\langle\Psi_{*}|\hat{T}| \Psi_{*}\right\rangle-\left\langle\Phi_{*}|\hat{T}| \Phi_{*}\right\rangle$ is nonnegative (128) and the second because $E_{C}$ is nonpositive. These two inequalities suggest that there always exists a $\gamma$ for which the correlation energy is exactly equal to the difference of the expectation values:

$$
E_{C}[n]=\left\langle\Psi_{*}\left|\hat{Y}_{\gamma}\right| \Psi_{*}\right\rangle-\left\langle\Phi_{*}\left|\hat{Y}_{\gamma}\right| \Phi_{*}\right\rangle .
$$

This definition of $\gamma$ stresses its density dependence. One can use Equation 39 to compute the value of $\gamma$ for the HEG, using the pair distribution function, for which good parameterizations are available (129). For the $\mathrm{SH}$, it is possible to obtain an analytical expression,

$$
\gamma(n)=1-3 n\left(\ln \left[-\varepsilon_{C}^{H E G}(n)\right]\right)^{\prime},
$$

while for RSHs the results are computed numerically.

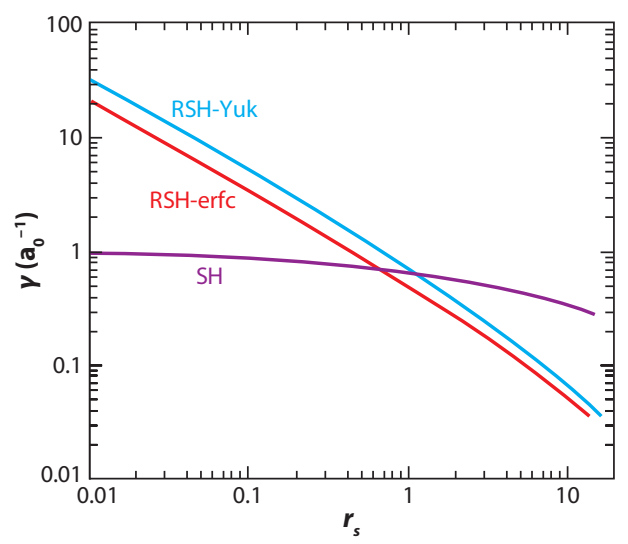

Figure 2

The value of $\gamma$ in the homogeneous electron gas as a function of the density parameter $r_{S}=(3 / 4 \pi n)^{1 / 3} \alpha_{0}^{-1}$ for the range-separated hybrid ( $\mathrm{RSH})$ and scaled-hybrid $(\mathrm{SH})$ interactions. 
The resulting dependency of $\gamma$ on the HEG density is shown in Figure 2 for the three functions of Equation 37. At large densities, $\varepsilon_{C}$ is dominated by kinetic energy correlation, so the value of $\gamma$ is large (reducing potential energy correlation), whereas for small densities, it is the potential energy correlations that are important so $\gamma$ is small. One noticeable feature in Figure 2 is the relatively mild change in $\gamma$ in the SH when compared to both RSHs. In the range $1<r_{s}<10$, corresponding to the chemically significant valence densities, $\gamma$ changes by approximately a factor of 2 in the $\mathrm{SH}$ and by more than a factor of 10 in the RSHs. Clearly, the correlation energy is more sensitive to $\gamma$ in RSHs than in SHs, indicating that pretuning $\gamma$ may be more important for RSHs than for SHs (although even in the latter this may be important; see 130).

The RSH with any finite value of $\gamma$ eliminates the important detrimental long-range problems in local and semilocal correlation-energy functionals. This was shown by Iikura et al. (44) in DFT and Tawada et al. (45) in TDDFT and has been subsequently confirmed by a series of works (46$53,131,132)$. In this section we demonstrate that a high level of performance can be achieved if one treats $\gamma$ as a system-dependent parameter tuned by ab initio considerations. All examples are based on the BNL functional described in Reference 52. This functional is given in Equations 25 and 26 with the following choices: $(a) y_{X C}^{\gamma}(r)$ is the RSH-erfc function (Equation 37), $(b) g_{X}^{\gamma}(n)$ is the LDA erf-exchange given in Reference 44, and $(c) g_{C}^{\gamma}(n,|\nabla n|)=g_{C}^{L Y P}(n,|\nabla n|)-w g_{X}^{\gamma}(n)$, based on the Lee-Yang-Parr (LYP) correlation functional (77) and where $w=0.1$ is a semiempirical constant (52).

Because $w \neq 0$, BNL does not retrace the HEG correlation energy for infinite homogeneous densities, but it does describe important properties of finite systems considerably better than when $w=0$ (52). The examples below show that BNL combined with $\gamma$ tuning (called BNL*) yields a balanced and usefully accurate description of systems that were often considered too difficult for DFT/TDDFT.

\section{Ionization Potentials}

The IP theorem (Equation 15) is an important connection between the $N$ electron and $N-1$ electron systems. Conventional DFT functionals and SHs grossly violate this condition, as seen in Figure 3: B3LYP HOMO energy is typically $70 \%$ of experimental vertical IPs. Despite this, the IPs are well estimated as B3LYP ground-state energy differences $E_{g s}(N)-E_{g s}(N-1)$ (mean
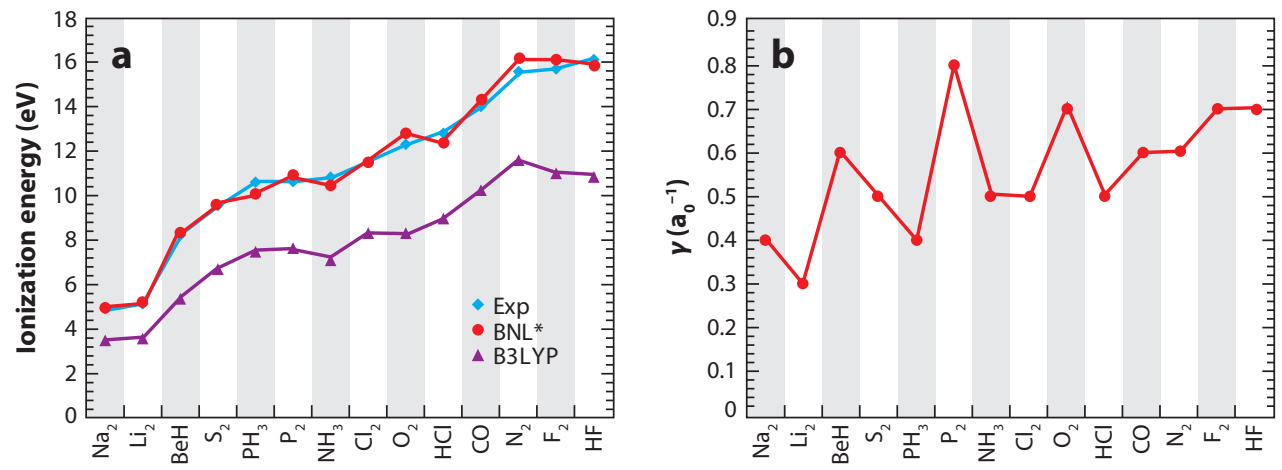

Figure 3

(a) The experimental vertical ionization energies versus calculated ionization potentials derived from B3LYP and BNL* HOMO energies for an assortment of small molecules. Basis set cc-pVTZ. (b) The values of the ab initio-motivated tuned range parameter $\gamma$ used for the BNL* calculation. Data based on Reference 52 . 


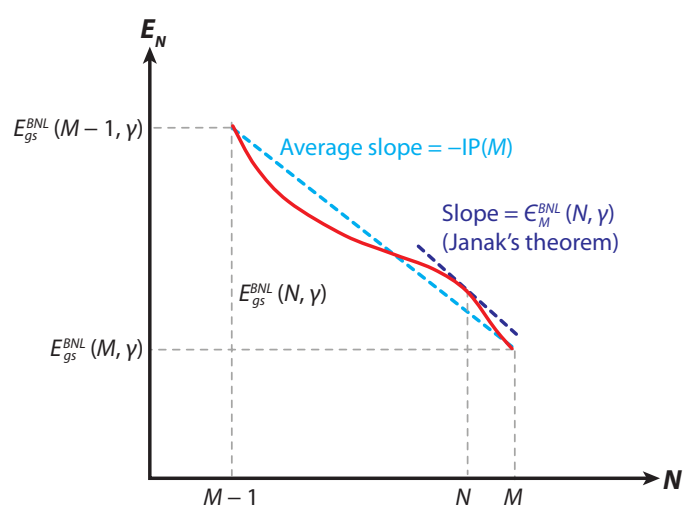

Figure 4

A schematic energy curve $E_{M}^{B N L}(N, \gamma)$. The dark blue slope at $N$ is $\varepsilon_{M}^{B N L}(N, \gamma)$ [Janak's theorem (67)], whereas the light blue $-\mathrm{IP}(M)$ is the average slope.

absolute deviance of $\sim 5 \%$ ). For BNL, we can enforce the IP theorem by tuning $\gamma$ to get

$$
\varepsilon_{N}^{B N L}(M, \gamma)=E_{g s}^{B N L}(M, \gamma)-E_{g s}^{B N L}(M-1, \gamma) .
$$

This should be done at some value of $N$ in the range $[M-1, M]$. When dealing with properties of the (integer) $M$ electron system, it is natural to take $N=M(52)$. Equation 41 requires that the slope of $E_{g s}^{B N L}(N, \gamma)$ as a function of $N$ be equal to the average slope between $N=M-1$ and $N=M$. This is shown in Figure 4, where a schematic curved line representing $E_{g s}^{B N L}(N, \gamma)$ is drawn in the $N-E$ plane connecting the points $\left(M-1, E_{g s}^{B N L}(M-1, \gamma)\right)$ and $\left(M, E_{g s}^{B N L}(M, \gamma)\right)$. The slope of this line is $\partial E_{g s}^{B N L} / \partial N$. By Janak's (67) theorem this slope is also equal to the HOMO energy $\varepsilon_{M}^{B N L}(N, \gamma)$. The exact $E_{g s}(N)$ curve must be a straight line (65) connecting the two end points in Figure 4. There is recent evidence of cases where RSH curves are in fact very close to straight lines (133).

In Figure 3 we find that $\gamma$ tuning according to Equation 41 leads to much improved IPs, with a mean absolute deviance of $2 \%$. We discuss another slope-motivated scheme below.

The $\gamma$-tuning procedure has an additional benefit: The occupied orbital energies become excellent approximations for inner negative IPs (ionization into excited states of the cation). This connects with long-standing controversies on the meaning of occupied orbital energies in DFT (60, 134-137). For example, Figure 5 presents the first four IPs of $\mathrm{H}_{2} \mathrm{O}$ spanning a range of 12 to $30 \mathrm{eV}$, calculated in two ways:

1. Koopmans': Set $\operatorname{IP}_{k}=-\varepsilon_{M-k+1}, k=1,2, \ldots$, where $M$ is the number of occupied orbitals and $\varepsilon_{m}$ is the $m$-th occupied orbital energy.

2. $\triangle \mathrm{SCF} / \mathrm{TD}$ : Compute the first $\mathrm{IP}\left(\mathrm{IP}_{1}\right)$ using a $\triangle \mathrm{SCF}$ procedure [i.e., the difference between self-consistent field (SCF) KS energies of the cation and the neutral]. Then set $\mathrm{IP}_{k}=$ $\mathrm{IP}_{1}+v_{k-1},(k=2,3, \ldots)$, where $v_{k}$ is the cation $k$-th excitation energy, calculated with TDDFT (or time-dependent HF).

Two remarkable findings are $(a)$ internal consistency, with the Koopmans' and $\triangle$ SCF/TD methods giving nearly the same result, and $(b)$ close proximity $(<3 \%)$ to experiment. The B3P86$30 \%$ SH [Becke exchange (79) mixed with $30 \%$ exact explicit exchange and Perdew's 86 correlation (138)] exhibits considerably larger errors of $-8 \%$ to $-20 \%$ (Koopmans) and +5 to $+7 \%$ $(\triangle \mathrm{SCF} / \mathrm{TD})$, whereas HF methods have similar errors with opposite signs. Statistical average of orbital potentials (SAOP) and exact KS Koopmans approaches (136) give good fits to experiment

SCF: self-consistent field 


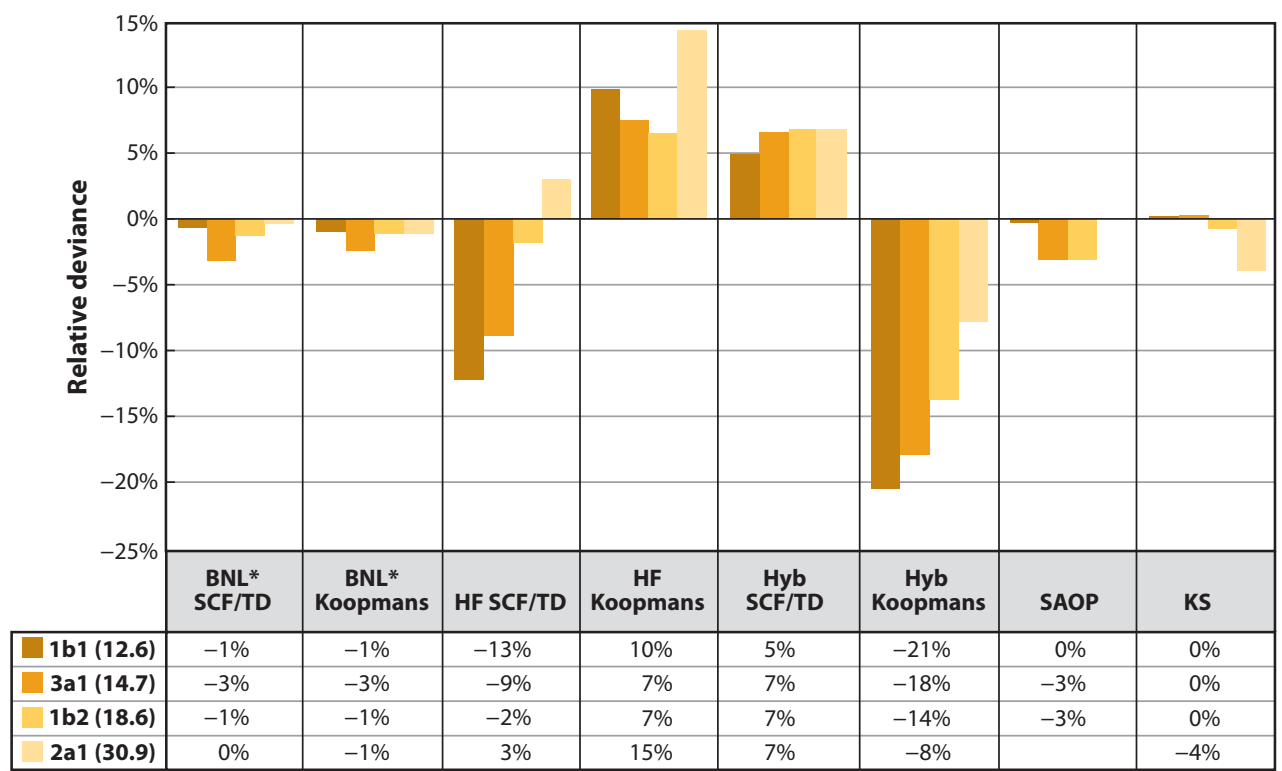

Figure 5

The relative deviance of ionization energy predictions in $\mathrm{H}_{2} \mathrm{O}$ from the first four experimental photoelectron ionization potentials (IPs) (139). The predictions are based on the $\gamma$-tuned BNL (BNL*) range-separated hybrid, Hartree-Fock (HF) approximation, scaled-hybrid (SH) B3P86-30\% (Hyb), SAOP results, and exact Kohn-Sham (KS) occupied orbital energies (the latter two are quoted from Reference 136). Koopmans' mean orbital energies are used. $\triangle \mathrm{SCF} / \mathrm{TD}$ means that $\triangle \mathrm{SCF}$ is used for the first IP, whereas the higher IPs are estimated by adding the relevant $\mathrm{H}_{2} \mathrm{O}^{+}$excitation energies calculated using time-dependent density functional theory.

as well. Similar findings hold also for $\mathrm{NH}_{3}, \mathrm{CH}_{2} \mathrm{O}$, and $\mathrm{HCOOH}$ and several organic molecules; details and partial theoretical elucidation will be published elsewhere.

\section{Symmetric Radical Cations}

One challenging prototype system for DFT methods is the symmetric radical cation dimer $\mathrm{A}_{2}^{+}$, where $A$ is any neutral molecule. $\mathrm{H}_{2}^{+}$is the simplest member of this family. Many chemically and biologically interesting processes, for example, the photoionization of the water dimer (140), involve the formation of such cations. Local, semilocal, and $\mathrm{SH}$ functionals fail to produce a reasonable BO potential energy surface (PES) for these molecules. The core of the problem is the nearly degenerate ground state when the monomer separation on $R$ is large: Because of selfrepulsion, these methods break the degeneracy and predict a unique charge-delocalized ground state. The uncorrected self-repulsion is dominant at long distances and the resulting PES is that of two repelling half-charged positive ions: $V_{B O}(R) \approx 2 \times E_{A}\left(M-\frac{1}{2}\right)+\frac{1}{4 R},(R \rightarrow \infty)$. When $A$ is an atom, the correct form of the asymptotic PES is the attractive atom-ion potential: $V_{B O}(R) \approx E_{A}(M)+E_{A}(M-1)-\frac{\alpha(A) e^{2}}{2 R^{4}}$, where $\alpha(A)$ is the polarizability of $A$.

The application of an RSH to this problem shows that it corrects the main deficiency of the PES, making it attractive instead of repulsive. However, the potential curve is still spurious in terms of its asymptotic value and its dependence on $R(53,141)$. In the asymptote, the relation 
$2 \times E_{A}\left(M-\frac{1}{2}\right)=E_{A}(M)+E_{A}(M-1)$ must hold (a special case of Equation 16). This can be achieved by tuning the parameter $\gamma$ so that

$$
\frac{1}{2}\left(E_{g s}^{B N L}\left(M-\frac{1}{2}, \gamma\right)-E_{g s}^{B N L}(M-1, \gamma)\right)=\frac{1}{2}\left(E_{g s}^{B N L}(M, \gamma)-E_{g s}^{B N L}\left(M-\frac{1}{2}, \gamma\right)\right) .
$$

This procedure of $\gamma$ tuning is different from the method based on the IP theorem, although in practice both methods give almost identical values of $\gamma$. Referring to Figure 4 and Equation 16, we see that Equation 42 is in effect demanding that the average slope of $E_{g s}(N)$ in the first halfinterval $\left[M-1, M-\frac{1}{2}\right]$ be equal to the average slope in the second half-interval $\left[M-\frac{1}{2}, M\right]$. The value of $\gamma$ found by the tuning procedure is strongly system-dependent. For $\mathrm{H}_{2}^{+} \gamma \rightarrow \infty$, and for $\mathrm{He}_{2}^{+}$and $\mathrm{Ne}_{2}^{+}, \gamma$ is $1.3 \mathrm{a}_{0}^{-1}$ and $0.9 \mathrm{a}_{0}{ }^{-1}$, respectively (141). With the tuned value of $\gamma$, the resulting potential exhibits many characteristics of the exact potential: The molecule-ion potential, $-\alpha(A) / 2 R^{4}$, is indeed obtained at large $R$; bond energies, bond lengths, and vibrational frequencies are also improved (141).

\section{Charge Transfer and Barriers}

DFT is often well-adapted for describing adsorbates on metals; many oxygen-metal systems do not exhibit an activation barrier in accordance with $\mathrm{KS}$ predictions. However, the $\mathrm{O}_{2}+\mathrm{Al}(111)$ sticking reaction is an exception: Experiments find an activation energy of $\sim 0.3-0.5 \mathrm{eV}(142)$ in contrast to the predictions of local and semilocal KS calculations (143). The strong attraction of $\mathrm{O}_{2}$ to the $\mathrm{Al}$ surface probably results from spurious partial electron transfer (similar to the $\mathrm{F}$...H system in Figure 1). One remedy is to impose spin restrictions on the $\mathrm{O}_{2}$ spin density (144), artificially favoring polarized spin density on the oxygen (electron transfer from the surface to the oxygen will lower this spin density). To analyze the system further, investigators examined the $\mathrm{O}_{2}+\mathrm{Al}_{5} \rightarrow \mathrm{O}_{2} \mathrm{Al}_{5}$ reaction using BNL and B3LYP, and indeed the barrier for the reaction is sensitive to self-repulsion. Figure 6 shows that the barrier predicted by the PBE (78) semilocal functional was considerably lower than that predicted by B3LYP/SH (84) and lower still than that predicted by BNL/RSH (145). Removing self-repulsion using RSHs is not practical for infinite
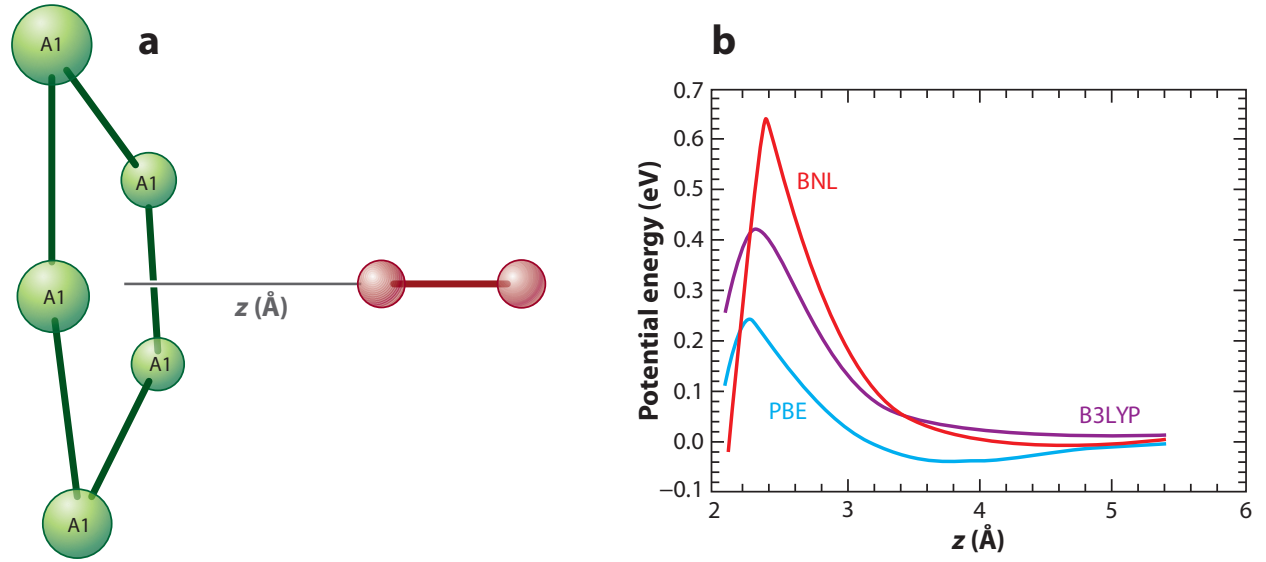

Figure 6

(a) The $\mathrm{O}_{2}+\mathrm{Al}_{5}$ system. The oxygen molecule approaches along the $\mathrm{z}$ coordinate in a perpendicular configuration. (b) The potential curve for the approaching oxygen molecule along the $z$ coordinate. Figure taken from Reference 145. 
metal surfaces: The long tail of exact exchange will likely cause spurious depletion of the density of states at the Fermi level.

\section{Charge-Transfer Excitations}

One strength of the TDGKS/RSH approach, as compared to the local/semilocal TDKS methods, is its ability to qualitatively account for long-range CTEs $(45,51,52,120,132,146,147)$. This is best analyzed in the TDGKS adiabatic linear-response equations, which can be written in an eigenvalue form we call the generalized Casida equations (101, 148, 149):

$$
\sum_{j s}\left(2 W_{k q, j s} \sqrt{\omega_{q k} \omega_{s j}}+\omega_{q k}^{2} \delta_{(q k)(s j)}\right) z_{s j}=\Omega_{A L R}^{2} z_{q k},
$$

where the indices refer to molecular spin orbitals: $q, s(j, k)$ are indices of unoccupied (occupied) molecular spin orbitals; $\omega_{q k}=\varepsilon_{q}-\varepsilon_{k}$; the eigenvalue $\Omega_{A L R}$ is the excitation energy; and the elements of the supermatrix $W$ are given by the double integrals

$$
\begin{aligned}
W_{q k, j}= & \iint d^{3} r d^{3} r^{\prime} \phi_{q}(\mathbf{r}) \phi_{s}\left(\mathbf{r}^{\prime}\right) \\
& \times\left[\left(\frac{1}{\left|\mathbf{r}-\mathbf{r}^{\prime}\right|}+f_{X C}^{\gamma}(n(\mathbf{r})) \delta\left(\mathbf{r}-\mathbf{r}^{\prime}\right)\right) \phi_{k}(\mathbf{r}) \phi_{j}\left(\mathbf{r}^{\prime}\right)-\bar{y}_{\gamma}\left(\left|\mathbf{r}-\mathbf{r}^{\prime}\right|\right) \phi_{k}\left(\mathbf{r}^{\prime}\right) \phi_{j}(\mathbf{r})\right],
\end{aligned}
$$

where $f_{X C}^{\gamma}(n)=\left(v_{X C}^{\gamma}(n)\right)^{\prime}$ is the XC kernel. The last term in the square brackets of Equation 44 is the long-range exchange, which eliminates the long-range self-repulsion in the Hartree term (the first term in the round parentheses). In long-range CTEs, the molecular spin orbitals are localized on two distant fragments: For example, $\phi_{k}$ is the HOMO (of the donor fragment $D$ and of the entire system) and $\phi_{q}$ is the LUMO (of the acceptor fragment $A$ and of the entire system as well). When the distance $R_{D A}$ is large, both the spatial overlap of these molecular spin orbitals and the exchange interaction become negligible; thus, the excitation energy is equal to the orbital-energy difference $\lim _{R_{D A} \rightarrow \infty} \Omega_{A L R}=\omega_{q k}$. When $R_{D A}$ is large but finite, the exchange term induces an additional $\frac{-1}{R_{D A}}$ term, and the adiabatic linear-response excitation energy is

$$
\Omega_{A L R}=\varepsilon_{q}-\varepsilon_{k}-\frac{1}{R_{D A}} \quad\left(R_{D A} \text { large }\right) .
$$

This result should be compared with Mulliken's law for CTEs:

$$
\Omega=\mathrm{IP}-\mathrm{EA}-\frac{1}{R_{D A}} \quad\left(R_{D A} \text { large }\right),
$$

where IP refers to the donor (or the system), and EA is the electron affinity of the acceptor (or the system). We note that in adiabatic TDKS, the matrix $W$ of Equation 44 does not have the explicit exchange term. Thus, the Casida equation based on TDKS has no source for the $-\frac{1}{R_{D A}}$ dependence required by Mulliken's law. Taking the difference between the two expressions, noting that $-\mathrm{IP}=\varepsilon_{k}$ by the IP theorem, we obtain

$$
\Omega-\Omega_{A L R}=-\varepsilon_{q}-\mathrm{EA}\left(R_{D A} \text { large }\right) .
$$

That $\Omega$ is different from $\Omega_{\mathrm{ALR}}$ is a fundamental problem of adiabatic linear-response TDDFT $(38,39,119)$, having common roots with the derivative discontinuity of Equation 19 . We have thus identified two separate problems with the application of adiabatic linear response to CTEs for large $R_{D A}$ as described in Table 1. In TDGKS/RSHs, the self-repulsion problem is overcome by the long-range explicit exchange terms, and the derivative-discontinuity problem is mitigated by proper tuning of $\gamma$. It is chosen to align as best as possible the donor IP with its HOMO 
Table 1 Problems with adiabatic linear response charge-transfer excitations at a large donor-acceptor distance, their source, and effects in various approximations

\begin{tabular}{l|l|l|l|l|l}
\hline & & \multirow{2}{*}{$\begin{array}{c}\text { Reason for } \\
\text { Violated condition }\end{array}$} & \multicolumn{3}{|c}{ Does the method avoid violation? } \\
\cline { 4 - 6 } & \multicolumn{1}{|c|}{$\begin{array}{c}\text { Tiolation } \\
\text { TDKS/local/sl }\end{array}$} & TDGKS/RSH & $\begin{array}{c}\text { TDGS/RSH + } \\
\text { tuning }\end{array}$ \\
\hline 1 & $R_{D A} \times\left[\Omega_{A L R}\left(R_{D A}\right)-\Omega_{A L R}(\infty)\right] \rightarrow-1$ & Self-repulsion & NO & YES & YES \\
\hline 2 & $\Omega_{A L R}(\infty)=I P(D)-E A(A)$ & $\begin{array}{c}\text { Derivative } \\
\text { discontinuity }\end{array}$ & NO & NO & Largely so \\
\hline
\end{tabular}

and the electron affinity of the acceptor with the HOMO of anionic acceptor (or, in a slightly different approach, the LUMO of the neutral acceptor). Reference 150 gives a fuller account of this approach, along with calculations of CTEs in aromatic-TCNE complexes, showing excellent results when compared to experiments.

\section{Rydberg Excitations}

The local and semilocal TDKS methods cannot describe Rydberg states because of the absence of the $-1 / r$ Coulomb tail in the average potential (see Figure 1). The RSH TDGKS approach does not suffer from this problem, as was first demonstrated in Reference 45. This was confirmed using the BNL/TDGKS approach (52), which showed excellent Rydberg excitation energies for several small molecules. However, the reported results were not good for the Rydberg excitations of benzene, which gave errors exceeding $0.5 \mathrm{eV}$ (52). We now show that this discrepancy is remedied by the usual procedure of tuning $\gamma$ (Equation 41). Figure 7 shows results with BNL and $\mathrm{BNL}^{*}$ compared to experiments. The $\mathrm{BNL}^{*}$ predictions follow all the experimental Rydberg excitations closely. Even the valence excitations are much improved. Thus, the tuning procedure is an essential element for achieving reliable quantitative predictions for Rydberg excitations using TDDTFT.

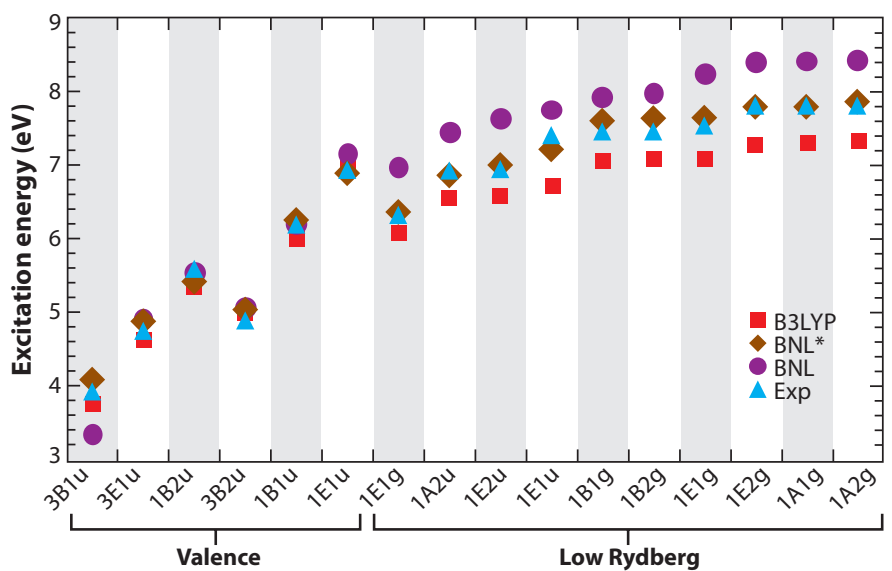

Figure 7

The time-dependent density functional theory valence and low Rydberg vertical excitation energies (eV) of benzene computed using B3LYP, BNL, and BNL* compared to experimental measurements (as taken from Reference 45). The basis set is daug-cc-pVTZ + an additional diffuse function. 


\section{SUMMARY}

This review discusses an approach to DFT that is based on the GKS theory and employs RSHs. Above we explain why this approach, in an adiabatic time-dependent context, can also be considered a TDDFT. We focus on the issue of ab initio-motivated schemes for range parameter tuning, using as guides the IP theorem and fractional electron-number exact results. Through examples and comparisons to experiment, we demonstrate how the tuning procedures considerably improve the scope and predictive power of DFT and TDDFT in several key applications.

The field is still open, as there are fundamental problems left unsolved. One pressing issue is that this approach lacks so-called size consistency: The energy and density of a system A placed far from a system $B$ are different from the energy of A when it is isolated because $\gamma_{A B}$ can be different from $\gamma_{A}$. Although this indeed is a severe problem, we note that the tuning of $\gamma$ can also help alleviate size consistency issues in local and semilocal functionals, such as the deleterious long-range charge sharing between atoms or the spurious energy curve for symmetric radical cation systems discussed above. An extreme example of the size-consistency problem is seen when A is a metal: Any $\gamma$ significantly different from zero might spuriously reduce the metallic density of states at the Fermi level. Another open subject is the derivative discontinuity that rises in longrange CTEs. The RSH approach mitigates this problem relative to SHs and local and semilocal KS methods; this issue requires additional study and testing. Finally, it seems that in TDDFT, any type of excitation will require a different choice of the $\gamma$ parameter, and it is not clear at present how such a parameter can be determined from first principles. From the study of band gaps in solids, there are indications that the dielectric constant of the various materials is an important component in determining $\gamma(151)$.

Despite these open issues, the examples shown here and ongoing research indicate that ab initio-motivated $\gamma$-tuning procedures offer a simple and practical approach for a high-quality description of the electronic structure of systems often considered too difficult for DFT.

\section{SUMMARY POINTS}

1. The RSH correlation energy can be formulated as a difference of interaction energies. This enables a formal definition of the range parameter (Equation 39).

2. The range parameter in the application of the RSH approach to HEG is more sensitive to density than the parameter in SHs (Figure 2).

3. Of the two methods discussed for $\gamma$ tuning based on the slopes of $E_{g s}(N)$ with respect to electron number $N$, one is based on the near-integer slope (IP theorem) and the other on a fractional electron-number slope. Both give nearly identical results.

4. An extended Runge-Gross theorem allows one to view time-dependent hybrid approaches as legitimate TDDFTs.

5. With regard to the adiabatic linear response for CTEs, in local/semilocal/SH timedependent approaches, the dependence of excitation energy on the donor-acceptor distance is spurious and the asymptotic excitations energies require a derivativediscontinuity correction. The time-dependent RSH approach fixes the dependency on distance, but the derivative discontinuity can still be very large unless $\gamma$ tuning is performed. It is notable that with such tuning the derivative discontinuity comes out very small. 
6. In the tuned RSH approach, the orbital energies are excellent approximations for inner IPs. Furthermore they agree well with the time-dependent RSH excitation energies of the cation (Figure 5).

7. Accurate Rydberg excitation energies are obtained by the tuned RSH approach (Figure 7).

8. Reaction barriers caused by charge transfer will probably be sensitive to spurious selfrepulsion, as seen for the $\mathrm{O}_{2}+\mathrm{Ag}_{5}$ system (Figure 6).

\section{DISCLOSURE STATEMENT}

The authors are not aware of any affiliations, memberships, funding, or financial holdings that might be perceived as affecting the objectivity of this review.

\section{ACKNOWLEDGMENTS}

We gratefully thank Professors Mel Levy and Leeor Kronik for helpful suggestions and corrections. This work was supported by a grant from the Israel Academy of Sciences and Humanities.

\section{LITERATURE CITED}

1. Parr RG, Yang W. 1989. Density Functional Theory of Atoms and Molecules. Oxford: Oxford Univ. Press

2. Dreizler RM, Gross EKU. 1990. Density Functional Theory: An Approach to the Quantum Many Body Problem. Berlin: Springer

3. Capelle K. 2006. A bird's-eye view of density-functional theory. Braz. 7. Phys. 36:1318-43

4. Koch W, Holthausen MC. 2001. A Chemist's Guide to Density Functional Theory. Heidelberg: Wiley

5. Gidopoulos NI, Wilson S. 2003. The Fundamentals of Electron Density, Density Matrix, and Density Functional Theory in Atoms, Molecules, and the Solid State. Dordrecht: Kluwer Academic

6. Seminario JM. 1996. Recent Developments and Applications of Modern Density Functional Theory. Amsterdam: Elsevier

7. Ellis DE. 1995. Density Functional Theory of Molecules, Clusters, and Solids. Dordrecht: Kluwer Academic

8. Dobson JF. 1999. Electron density functional theory. Int. F. Mod. Phys. B 13:511-23

9. Siegbahn PEM, Blomberg MRA. 1999. Density functional theory of biologically relevant metal centers. Annu. Rev. Phys. Chem. 50:221-49

10. Van Doren VE, Van Alsenoy C, Geerlings P. 2001. Density Functional Theory and its Application to Materials: Antwerp, Belgium, 8-10 fune 2000. Melville, NY: Am. Inst. Phys.

11. Bencini A, Totti F, Daul CA, Doclo K, Fantucci P, Barone V. 1997. Density functional calculations of magnetic exchange interactions in polynuclear transition metal complexes. Inorg. Chem. 36:5022-30

12. Kohn W, Sham LJ. 1965. Self-consistent equations including exchange and correlation effects. Phys. Rev. 140:A1133

13. Seidl A, Gorling A, Vogl P, Majewski JA, Levy M. 1996. Generalized Kohn-Sham schemes and the band-gap problem. Phys. Rev. B 53:3764-74

14. Ovchinnikov IV, Bartell LA, Neuhauser D. 2007. Hydrodynamic tensor density functional theory with correct susceptibility. F. Chem. Phys. 126:1341019

15. Wang YA, Govind N, Carter EA. 1998. Orbital-free kinetic-energy functionals for the nearly free electron gas. Phys. Rev. B 58:13465-71

16. Ovchinnikov IV, Neuhauser D. 2006. Orbital-free tensor density functional theory. 7. Chem. Phys. 124:024105

17. Runge E, Gross EKU. 1984. Density functional theory for time dependent systems. Phys. Rev. Lett. $52: 997-1000$ 
18. Marques M, Rubio A, Ullrich CA, Burke K, Nogueira F, Gross A. 2006. Time-Dependent Density Functional Theory. Berlin: Springer

19. Ullrich CA, Gossmann UJ, Gross EKU. 1995. Density-functional approach to atoms in strong laser pulses. Phys. Chem. Chem. Phys. 99:488-97

20. Petersilka M, Gross EKU, Burke K. 2000. Excitation energies from time-dependent density functional theory using exact and approximate potentials. Int. 7. Quant. Chem. 80:534-54

21. Furche F, Ahlrichs R. 2002. Adiabatic time-dependent density functional methods for excited state properties. F. Chem. Phys. 117:7433-47

22. Baer R. 2002. Nonadiabatic couplings from time dependent density functional theory. Chem. Phys. Lett. 364:75-79

23. Baer R, Neuhauser D, Zdanska P, Moiseyev N. 2003. Ionization and high-harmonic generation in aligned benzene by intense circularly polarized light. Phys. Rev. A 68:043406

24. Martinez TJ. 2003. Photochemistry and excited state dynamics with time-dependent density functional theory. Abstr. Pap. Am. Chem. Soc. 225:U707

25. Baer R, Weiss S, Neuhauser D. 2004. Enhanced absorption induced by a metallic nanoshell. Nano Lett. 4:85-88

26. Castro A, Marques MAL, Alonso JA, Bertsch GF, Rubio A. 2004. Excited states dynamics in timedependent density functional theory: high-field molecular dissociation and harmonic generation. Eur. Phys. 7. D 28:211-18

27. Craig CF, Duncan WR, Prezhdo OV. 2005. Trajectory surface hopping in the time-dependent KohnSham approach for electron-nuclear dynamics. Phys. Rev. Lett. 95:163001

28. Kurth S, Stefanucci G, Almbladh CO, Rubio A, Gross EKU. 2005. Time-dependent quantum transport: a practical scheme using density functional theory. Phys. Rev. B 72:035308

29. Livshits E, Baer R. 2006. Time-dependent density-functional studies of the D2 Coulomb explosion. 7 . Phys. Chem. A 110:8443-50

30. Salzner U. 2007. Theoretical investigation of excited states of oligothiophenes and of their monocations. 7. Chem. Theor. Comp. 3:1143-57

31. Andzelm J, Rawlett AM, Orlicki JA, Snyder JF. 2007. Optical properties of phthalocyanine and naphthalocyanine compounds. F. Chem. Theor. Comp. 3:870-77

32. Prociuk A, Dunietz BD. 2008. Modeling time-dependent current through electronic open channels using a mixed time-frequency solution to the electronic equations of motion. Phys. Rev. B 78:165112-16

33. Gavnholt J, Rubio A, Olsen T, Thygesen KS, Schiotz J. 2009. Hot-electron-assisted femtochemistry at surfaces: a time-dependent density functional theory approach. Phys. Rev. B 79:195405-10

34. Baer R, Seideman T, Ilani S, Neuhauser D. 2004. Ab initio study of the alternating current impedance of a molecular junction. 7. Chem. Phys. 120:3387-96

35. Cohen AJ, Mori-Sanchez P, Yang WT. 2008. Insights into current limitations of density functional theory. Science 321:792-94

36. Zhang Y, Yang W. 1998. A challenge for density functionals: self-interaction error increases for systems with a noninteger number of electrons. F. Chem. Phys. 109:2604-8

37. Champagne B, Perpete EA, van Gisbergen SJA, Baerends E-J, Snijders JG, et al. 1998. Assessment of conventional density functional schemes for computing the polarizabilities and hyperpolarizabilities of conjugated oligomers: an ab initio investigation of polyacetylene chains. F. Chem. Phys. 109:10489-98

38. Dreuw A, Weisman JL, Head-Gordon M. 2003. Long-range charge-transfer excited states in timedependent density functional theory require non-local exchange. F. Am. Chem. Phys. 119:2943-46

39. Autschbach J. 2009. Charge-transfer excitations and time-dependent density functional theory: problems and some proposed solutions. Chemphyschem 10:1757-60

40. van Leeuwen R, Baerends EJ. 1994. Exchange-correlation potential with correct asymptotic behavior. Phys. Rev. A 49:2421-31

41. Tozer DJ, Handy NC. 1998. Improving virtual Kohn-Sham orbitals and eigenvalues: application to excitation energies and static polarizabilities. F. Chem. Phys. 109:10180-89

42. Ullrich CA, Gossmann UJ, Gross EKU. 1995. Time-dependent optimized effective potential. Phys. Rev. Lett. 74:872-75 
43. Savin A. 1995. Recent Advances in Density Functional Methods Part I, ed. DP Chong. Singapore: World Sci.

44. Iikura H, Tsuneda T, Yanai T, Hirao K. 2001. A long-range correction scheme for generalized-gradientapproximation exchange functionals. 7. Chem. Phys. 115:3540-44

45. Tawada Y, Tsuneda T, Yanagisawa S, Yanai T, Hirao K. 2004. A long-range-corrected time-dependent density functional theory. F. Chem. Phys. 120:8425-33

46. Yanai T, Tew DP, Handy NC. 2004. A new hybrid exchange-correlation functional using the Coulombattenuating method (CAM-B3LYP). Chem. Phys. Lett. 393:51-57

47. Baer R, Neuhauser D. 2005. A density functional theory with correct long-range asymptotic behavior. Phys. Rev. Lett. 94:043002

48. Gerber IC, Angyan JG. 2005. Hybrid functional with separated range. Chem. Phys. Lett. 415:100-5

49. Zhao Y, Truhlar DG. 2006. Density functional for spectroscopy: no long-range self-interaction error, good performance for Rydberg and charge-transfer states, and better performance on average than B3LYP for ground states. 7. Phys. Chem. A 110:13126-30

50. Vydrov OA, Scuseria GE. 2006. Assessment of a long-range corrected hybrid functional. F. Chem. Phys. 125:234109

51. Peach MJG, Helgaker T, Salek P, Keal TW, Lutnaes OB, et al. 2006. Assessment of a Coulombattenuated exchange-correlation energy functional. Phys. Chem. Chem. Phys. 8:558-62

52. Livshits E, Baer R. 2007. A well-tempered density functional theory of electrons in molecules. Phys. Chem. Chem. Phys. 9:2932-41

53. Chai JD, Head-Gordon M. 2008. Systematic optimization of long-range corrected hybrid density functionals. F. Chem. Phys. 128:084106

54. Mori-Sanchez P, Cohen AJ, Yang WT. 2006. Self-interaction-free exchange-correlation functional for thermochemistry and kinetics. F. Chem. Phys. 124:09112

55. Hohenberg P, Kohn W. 1964. Inhomogeneous electron gas. Phys. Rev. 136:B864

56. Levy M. 1979. Universal variational functionals of electron densities, first-order density matrices, and natural spin-orbitals and solution of the V-representability problem. Proc. Natl. Acad. Sci. USA 76:606265

57. Almbladh C-O, von Barth U. 1985. Exact results for the charge and spin densities, exchange-correlation potentials, and density-functional eigenvalues. Phys. Rev. B 31:3231-44

58. Handy NC, Marron MT, Silverst HJ. 1969. Long-range behavior of Hartree-Fock orbitals. Phys. Rev. 180:45-48

59. Levy M, Perdew JP. 1983. Density Functional Methods in Physics, ed. R Dreizler, J Perovidencia. New York: Plenum

60. Kümmel S, Kronik L. 2008. Orbital-dependent density functionals: theory and applications. Rev. Mod. Phys. 80:3-60

61. Gorling A. 2005. Orbital- and state-dependent functionals in density-functional theory. F. Chem. Phys. 123:062203

62. Engel E. 2003. Orbital-dependent functionals for the exchange-correlation energy: a third generation of density functionals. In A Primer in Density Functional Theory, ed. C Fiolhais, F Nogueira, M Marques, pp. 56-122. New York: Springer

63. Katriel J, Davidson ER. 1980. Asymptotic behavior of atomic and molecular wave-functions. Proc. Natl. Acad. Sci. USA 77:4403-6

64. Levy M, Perdew JP, Sahni V. 1984. Exact differential equation for the density and ionization energy of a many-particle system. Phys. Rev. A 30:2745-48

65. Perdew JP, Parr RG, Levy M, Balduz JL. 1982. Density functional theory for fractional particle number: derivative discontinuities of the energy. Phys. Rev. Lett. 49:1691-94

66. Valone SM. 1980. Consequences of extending 1-matrix energy functionals from pure-state representable to all ensemble representable 1 matrices. 7. Chem. Phys. 73:1344-49

67. Janak J. 1978. Proof that $\partial E / \partial n_{i}=\varepsilon$ in density-functional theory. Phys. Rev. B 18:7165-68

68. Giuliani G, Vignale G. 2005. Quantum Theory of the Electron Liquid. Cambridge, UK: Cambridge Univ. Press 
69. Ceperley DM, Alder BJ. 1980. Ground state of the electron gas by a stochastic model. Phys. Rev. Lett. 45:566-69

70. Perdew JP, Wang Y. 1992. Accurate and simple analytic representation of the electron-gas correlation energy. Phys. Rev. B 45:13244-49

71. Perdew JP, Zunger A. 1981. Self-interaction correction to density functional theory approximations for many electron systems. Phys. Rev. B 23:5048-79

72. Merkle R, Savin A, Preuss H. 1992. Singly ionized first-row dimers and hydrides calculated with the fully numerical density-functional program NUMOL. F. Chem. Phys. 97:9216-21

73. van Faassen M, de Boeij PL, van Leeuwen R, Berger JA, Snijders JG. 2002. Ultranonlocality in time-dependent current-density-functional theory: application to conjugated polymers. Phys. Rev. Lett. $88: 186401$

74. Mori-Sanchez P, Cohen AJ, Yang WT. 2006. Many-electron self-interaction error in approximate density functionals. F. Chem. Phys. 125:201102

75. Kümmel S, Kronik L, Perdew JP. 2004. Electrical response of molecular chains from density functional theory. Phys. Rev. Lett. 93:213002

76. Ruzsinszky A, Perdew JP, Csonka GI, Vydrov OA, Scuseria GE. 2007. Density functionals that are oneand two- are not always many-electron self-interaction-free, as shown for $\mathrm{H}_{2}{ }^{+}, \mathrm{He}_{2}{ }^{+}, \mathrm{LiH}^{+}$, and $\mathrm{Ne}_{2}{ }^{+}$. 7. Chem. Phys. 126:104102

77. Lee CT, Yang WT, Parr RG. 1988. Development of the Colle-Salvetti correlation-energy formula into a functional of the electron density. Phys. Rev. B 37:785-89

78. Perdew JP, Burke K, Ernzerhof M. 1996. Generalized gradient approximation made simple. Phys. Rev. Lett. 77:3865-68

79. Becke AD. 1988. Density-functional exchange-energy approximation with correct asymptotic behavior. Phys. Rev. A 38:3098-100

80. Lee C, Zhou Z. 1991. Exchange-energy density functional: reparametrization of Becke's formula and derivation of second-order gradient correction. Phys. Rev. A 44:1536-39

81. Becke AD. 1993. A new mixing of Hartree-Fock and local density-functional theories. F. Chem. Phys. 98:1372-77

82. Adamo C, Scuseria GE, Barone V. 1999. Accurate excitation energies from time-dependent density functional theory: assessing the PBE0 model. F. Chem. Phys. 111:2889-99

83. Adamo C, Barone V. 1998. Exchange functionals with improved long-range behavior and adiabatic connection methods without adjustable parameters: the mPW and mPW1PW models. F. Chem. Phys. 108:664-75

84. Becke AD. 1993. Density-functional thermochemistry. 3. The role of exact exchange. 7. Chem. Phys. 98:5648-52

85. Perdew JP, Ernzerhof M, Burke K. 1996. Rationale for mixing exact exchange with density functional approximations. 7. Chem. Phys. 105:9982-85

86. Barone V. 1994. Inclusion of Hartree-Fock exchange in the density-functional approach: benchmark computations for diatomic molecules containing H, B, C, N, O, and F atoms. Chem. Phys. Lett. 226:39298

87. Zhao Y, Lynch BJ, Truhlar DG. 2004. Development and assessment of a new hybrid density functional model for thermochemical kinetics. F. Phys. Chem. A 108:2715-19

88. Leininger T, Stoll H, Werner H-J, Savin A. 1997. Combining long-range configuration interaction with short-range density functionals. Chem. Phys. Lett. 275:151-60

89. Akinaga Y, Ten-no S. 2008. Range-separation by the Yukawa potential in long-range corrected density functional theory with Gaussian-type basis functions. Chem. Phys. Lett. 462:348-51

90. Robinson JE, Bassani F, Knox RS, Schreiffer JR. 1962. Screening correction to the Slater exchange potential. Phys. Rev. Lett. 9:215-17

91. Krukau AV, Scuseria GE, Perdew JP, Savin A. 2008. Hybrid functionals with local range separation. 7 . Chem. Phys. 129:124103

92. van Leeuwen R. 1998. Causality and symmetry in time-dependent density-functional theory. Phys. Rev. Lett. 80:1280-83 
93. Thiele M, Gross EKU, Kümmel S. 2008. Adiabatic approximation in nonperturbative time-dependent density-functional theory. Phys. Rev. Lett. 100:153004

94. Baer R. 2009. Prevalence of the adiabatic exchange-correlation potential approximation in timedependent density functional theory. Theochem. 7. Mol. Struct. 914:19-21

95. Ando T. 1977. Inter-subband optical absorption in space-charge layers on semiconductor surfaces. $Z$. Phys. B 26:263-66

96. Zangwill A, Soven P. 1980. Density-functional approach to local-field effects in finite systems: photoabsorption in the rare gases. Phys. Rev. A 21:1561-72

97. Petersilka M, Gossmann UJ, Gross EKU. 1996. Excitation energies from time-dependent densityfunctional theory. Phys. Rev. Lett. 76:1212-15

98. Casida ME, Jamorski C, Casida KC, Salahub DR. 1998. Molecular excitation energies to high-lying bound states from time-dependent density-functional response theory: characterization and correction of the time-dependent local density approximation ionization threshold. F. Chem. Phys. 108:4439-49

99. Yabana K, Bertsch GF. 1999. Time-dependent local-density approximation in real time: application to conjugated molecules. Int. 7. Quant. Chem. 75:55-66

100. Hsu CP, Hirata S, Head-Gordon M. 2001. Excitation energies from time-dependent density functional theory for linear polyene oligomers: butadiene to decapentaene. F. Phys. Chem. A 105:451-58

101. Hirata S, Lee TJ, Casida ME. 1996. Time-dependent density functional response theory of molecular systems: theory, computational methods, and functionals. In Recent Developments and Applications in Density Functional Theory, ed. JM Seminario, pp. 391-439. Amsterdam: Elsevier

102. Chelikowsky JR, Kronik L, Vasiliev I. 2003. Time-dependent density-functional calculations for the optical spectra of molecules, clusters, and nanocrystals. 7. Phys. Condens. Mater. 15:R1517

103. Gross EKU, Kohn W. 1985. Local density-functional theory of frequency-dependent linear response. Phys. Rev. Lett. 55:2850-52

104. Maitra NT, Burke K, Woodward C. 2002. Memory in time-dependent density functional theory. Phys. Rev. Lett. 89:023002

105. Maitra NT, Zhang F, Cave RJ, Burke K. 2004. Double excitations within time-dependent density functional theory linear response. 7. Chem. Phys. 120:5932-37

106. Kurzweil Y, Baer R. 2006. Quantum memory effects on the dynamics of electrons in gold clusters. Phys. Rev. B 73:075413

107. Dobson JF. 1994. Harmonic-potential theorem: implications for approximate many-body theories. Phys. Rev. Lett. 73:2244-47

108. Vignale G. 1995. Center-of-mass and relative motion in time-dependent density-functional theory. Phys. Rev. Lett. 74:3233-36

109. Vignale G, Kohn W. 1996. Current-dependent exchange-correlation potential for dynamical linear response theory. Phys. Rev. Lett. 77:2037-40

110. van Leeuwen R. 2001. Key concepts in time-dependent density-functional theory. Int. F. Mod. Phys. B 15:1969-2023

111. Dobson JF, Bunner MJ, Gross EKU. 1997. Time-dependent density functional theory beyond linear response: an exchange-correlation potential with memory. Phys. Rev. Lett. 79:1905-8

112. Kurzweil Y, Baer R. 2004. Time-dependent exchange-correlation current density functionals with memory. 7. Chem. Phys. 121:8731-41

113. Kurzweil Y, Baer R. 2005. Genereic Galilean-invariant exchange-correlation functionals with quantum memory. Phys. Rev. B 72:035106

114. Tokatly IV. 2005. Quantum many-body dynamics in a Lagrangian frame: I. Equations of motion and conservation laws. Phys. Rev. B 71:165104

115. Ullrich CA, Tokatly IV. 2006. Nonadiabatic electron dynamics in time-dependent density-functional theory. Phys. Rev. B 73:235102

116. Baer R, Kurzweil Y, Cederbaum LS. 2005. Time-dependent density functional theory for non-adiabatic processes. Isr. F. Chem. 45:161-70

117. Kurzweil Y, Baer R. 2008. Adapting approximate memory potentials for time-dependent density functional theory. Phys. Rev. B 77:085121 
118. Kurzweil Y, Head-Gordon M. 2009. Improving approximate-optimized effective potentials by imposing exact conditions: theory and applications to electronic statics and dynamics. Phys. Rev. A 80:012509

119. Tozer DJ. 2003. Relationship between long-range charge-transfer excitation energy error and integer discontinuity in Kohn-Sham theory. F. Chem. Phys. 119:12697-99

120. Stein T, Kronik L, Baer R. 2009. Reliable prediction of charge transfer excitations in molecular complexes using time-dependent density functional theory. 7. Am. Chem. Soc. 131:2818-20

121. Mundt M, Kümmel S. 2006. Optimized effective potential in real time: problems and prospects in time-dependent density-functional theory. Phys. Rev. A 74:022511

122. Krieger JB, Li Y, Iafrate GJ. 1992. Construction and application of an accurate local spin-polarized Kohn-Sham potential with integer discontinuity: exchange-only theory. Phys. Rev. A 45:101-6

123. Chu X, Chu SI. 2001. Time-dependent density-functional theory for molecular processes in strong fields: study of multiphoton processes and dynamical response of individual valence electrons of $\mathrm{N}_{2}$ in intense laser fields. Phys. Rev. A 64:063404

124. Mundt M, Kummel S, van Leeuwen R, Reinhard PG. 2007. Violation of the zero-force theorem in the time-dependent Krieger-Li-Iafrate approximation. Phys. Rev. A 75:133004

125. Langreth DC, Perdew JP. 1975. The exchange-correlation energy of a metallic surface. Solid State Comm. 17:1425-29

126. Ernzerhof M, Perdew JP, Burke K. 1997. Coupling-constant dependence of atomization energies. Int. 7. Quant. Chem. 64:285-95

127. Cohen AJ, Mori-Sanchez P, Yang WT. 2007. Assessment and formal properties of exchange-correlation functionals constructed from the adiabatic connection. 7. Chem. Phys. 127:034101

128. Levy M, Perdew JP. 1985. Hellmann-Feynman, virial, and scaling requisites for the exact universal density functionals: shape of the correlation potential and diamagnetic susceptibility for atoms. Phys. Rev. A 32:2010-21

129. Gori-Giorgi P, Perdew JP. 2002. Pair distribution function of the spin-polarized electron gas: a firstprinciples analytic model for all uniform densities. Phys. Rev. B 66:165118

130. Lynch BJ, Truhlar DG. 2001. How well can hybrid density functional methods predict transition state geometries and barriers? 7. Phys. Chem. A 105:2936-41

131. Baer R, Livshits E, Neuhauser D. 2006. Avoiding self-repulsion in density functional description of biased molecular junctions. Chem. Phys. 329:266-75

132. Jacquemin D, Perpete EA, Scuseria GE, Ciofini I, Adamo C. 2008. TD-DFT performance for the visible absorption spectra of organic dyes: conventional versus long-range hybrids. F. Chem. Theor. Comp. 4:123-35

133. Cohen AJ, Mori-Sanchez P, Yang WT. 2007. Development of exchange-correlation functionals with minimal many-electron self-interaction error. f. Chem. Phys. 126:191109

134. Perdew JP, Norman MR. 1982. Electron removal energies in Kohn-Sham density-functional theory. Phys. Rev. B 26:5445-50

135. Perdew JP, Levy M. 1983. Physical content of the exact Kohn-Sham orbital energies: band gaps and derivative discontinuities. Phys. Rev. Lett. 51:1884-87

136. Chong DP, Gritsenko OV, Baerends EJ. 2002. Interpretation of the Kohn-Sham orbital energies as approximate vertical ionization potentials. 7. Chem. Phys. 116:1760-72

137. Korzdorfer T, Kümmel S, Marom N, Kronik L. 2009. When to trust photoelectron spectra from KohnSham eigenvalues: the case of organic semiconductors. Phys. Rev. B 79:201205

138. Perdew JP. 1986. Density-functional approximation for the correlation energy of the inhomogeneous electron gas. Phys. Rev. B 33:8822-24

139. Potts AW, Price WC. 1972. Photoelectron spectra and valence shell orbital structures of groups V and VI hydrides. Proc. R. Soc. Lond. A 326:181-87

140. Pieniazek PA, VandeVondele J, Jungwirth P, Krylov AI, Bradforth SE. 2008. Electronic structure of the water dimer cation. 7. Phys. Chem. A 112:6159-70

141. Livshits E, Baer R. 2008. A density functional theory for symmetric radical cations from bonding to dissociation. F. Phys. Chem. A 112:12789-91

142. Osterlund L, Zoric I, Kasemo B. 1997. Dissociative sticking of $\mathrm{O}_{2}$ on $\mathrm{Al}(111)$. Phys. Rev. B 55:15452-55 
143. Yourdshahyan Y, Razaznejad B, Lundqvist BI. 2001. Adiabatic potential-energy surface of $\mathrm{O}_{2} / \mathrm{Al}(111)$ : rare entrance-channel barriers but molecularly chemisorbed state apt for abstraction. Solid State Comm. 117:531-35

144. Behler J, Delley B, Lorenz S, Reuter K, Scheffler M. 2005. Dissociation of $\mathrm{O}_{2}$ at $\mathrm{Al}(111)$ : the role of spin selection rules. Phys. Rev. Lett. 94:036104

145. Livshits E, Baer R, Kosloff R. 2009. The deleterious effects of long-range self-repulsion on the density functional description of $\mathrm{O}_{2}$ sticking on aluminum. 7. Phys. Chem. A 113:7521-27

146. Govind N, Valiev M, Jensen L, Kowalski K. 2009. Excitation energies of zinc porphyrin in aqueous solution using long-range corrected time-dependent density functional theory. F. Phys. Chem. A 113:6041-43

147. Andzelm J, Rinderspacher BC, Rawlett A, Dougherty J, Baer R, Govind N. 2009. Performance of DFT methods in the calculation of optical spectra of TCF-chromophores. F. Chem. Theor. Comp. 5:2835-46

148. Jamorski C, Casida ME, Salahub DR. 1996. Dynamic polarizabilities and excitation spectra from a molecular implementation of time-dependent density-functional response theory: $\mathrm{N}_{2}$ as a case study. $\mathcal{F}$. Chem. Phys. 104:5134-47

149. Bauernschmitt R, Ahlrichs R. 1996. Treatment of electronic excitations within the adiabatic approximation of time dependent density functional theory. Chem. Phys. Lett. 256:454-64

150. Burke K, Werschnik J, Gross EKU. 2005. Time-dependent density functional theory: past, present, and future. 7. Chem. Phys. 123:062206

151. Eisenberg HS, Baer R. 2009. A new generalized Kohn-Sham method for fundamental band gaps in solids. Phys. Chem. Chem. Phys. 11:4674-80 
争

Annual Review of Physical Chemistry

Contents

Volume 61, 2010

On Walking in the Footprints of Giants

Marilyn E. Facox ...................................................... 1

Novel Computational Methods for Nanostructure Electronic

Structure Calculations

Lin-Wang Wang

Hyper-Raman Scattering by Molecular Vibrations

Anne Myers Kelley. ...

Chemistry of Hofmeister Anions and Osmolytes

Yanjie Zhang and Paul S. Cremer...

Tuned Range-Separated Hybrids in Density Functional Theory

Roi Baer, Ester Livshits, and Ulrike Salzner

Subcellular Dynamics and Protein Conformation Fluctuations

Measured by Fourier Imaging Correlation Spectroscopy

Eric N. Senning and Andrew H. Marcus

Oxide Surface Science

Ulrike Diebold, Shao-Chun Li, and Michael Scbmid ...

The Diabatic Picture of Electron Transfer, Reaction Barriers, and

Molecular Dynamics

Troy Van Voorhis, Tim Kowalczyk, Benjamin Kaduk, Lee-Ping Wang,

Chiao-Lun Cheng, and Qin Wu

Electrostatics of Strongly Charged Biological Polymers: Ion-Mediated

Interactions and Self-Organization in Nucleic Acids and Proteins

Gerard C.L. Wong and Lois Pollack

Dynamics on the Way to Forming Glass: Bubbles in Space-Time

David Chandler and Juan P. Garraban

Functional Motifs in Biochemical Reaction Networks

Jobn 7. Tyson and Béla Novák 
Electronic Properties of Nonideal Nanotube Materials: Helical

Symmetry Breaking in DNA Hybrids

Slava V. Rotkin

Molecular Structural Dynamics Probed by Ultrafast X-Ray Absorption

Spectroscopy

Christian Bressler and Majed Chergui

Statistical Mechanical Concepts in Immunology

Arup K. Chakraborty and Andrej Košmrlj

Biological Cluster Mass Spectrometry

Nicholas Winograd and Barbara 7. Garrison 305

Bio-Enabled Synthesis of Metamaterials

Christopher C. DuFort and Bogdan Dragnea

Superresolution Imaging using Single-Molecule Localization

George Patterson, Michael Davidson, Suliana Manley, and Fennifer Lippincott-Schwartz

From Artificial Atoms to Nanocrystal Molecules: Preparation and

Properties of More Complex Nanostructures

Charina L. Choi and A. Paul Alivisatos

Transition-Path Theory and Path-Finding Algorithms for the Study of Rare Events

Weinan E and Eric Vanden-Eijnden

Complex Fluids: Probing Mechanical Properties of Biological Systems with Optical Tweezers

H. Daniel Ou-Yang and Ming-Tzo Wei ...

Enhanced Sampling of Nonequilibrium Steady States

Alex Dickson and Aaron R. Dinner

Fluctuations in Biological and Bioinspired Electron-Transfer Reactions

Spiros S. Skourtis, David H. Waldeck, and David N. Beratan 461

\section{Indexes}

Cumulative Index of Contributing Authors, Volumes 57-61 .................... 487

Cumulative Index of Chapter Titles, Volumes 57-61 490

\section{Errata}

An online log of corrections to Annual Review of Physical Chemistry articles may be found at http://physchem.annualreviews.org/errata.shtml 